University of Redlands

\title{
Application of Satellite Imagery for Optimal Sample Selection and Vineyard Yield Estimation
}

A Major Individual Project submitted in partial satisfaction of the requirements for the degree of Master of Science in Geographic Information Systems

\author{
by \\ Brett Wong \\ Ruijin Ma, Ph.D., Committee Chair \\ Fang Ren, Ph.D.
}

August 2020 
Application of Satellite Imagery for Optimal Sample Selection and Vineyard Yield Estimation

Copyright $\odot 2020$

by

Brett Wong 
The report of Brett Wong is approved.

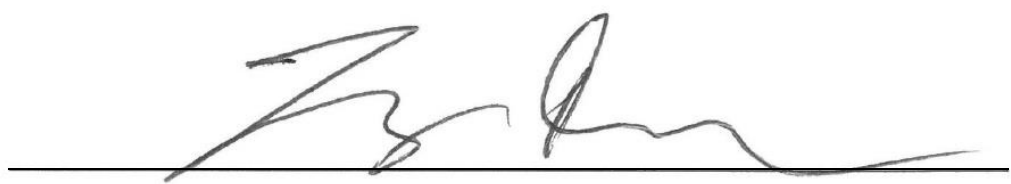

Fang Ren, Ph.D.

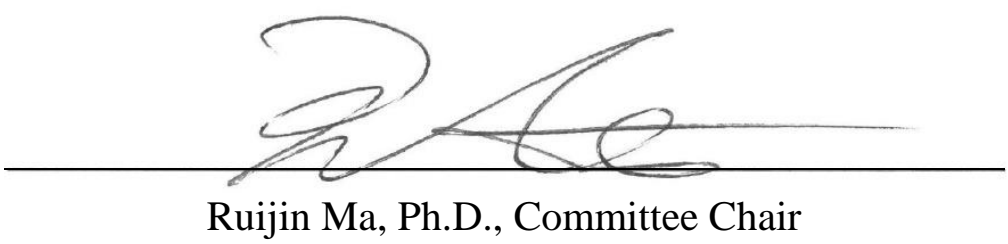

August 2020 



\section{Acknowledgements}

The completion of this project would not have been possible without the support of many people. First, I would like to thank the faculty and staff at the University of Redland's Department of Geographic Information Science. I would like to give a special thanks to Ruijin Ma for being my advisor and for providing guidance throughout this project. I would also like to express my gratitude to Fang Ren for the statistical analysis advice, Douglas Flewelling for the database knowledge, and Mark Kumler for the poster feedback. Thank you to the Esri faculty of Charlie Frye, Ken Baloun, and Jonathan Quinn for their courses and industry knowledge that have been provided. Also, I would like to show my gratitude to Nicole Kanahele-Stutz for her edits during the writing process of this project. Last but definitely not least, thank you Andrea Alvarado for all of the help you have provided throughout my time as a student, and for the endless supply of candy at your desk.

I would like to thank my friends and family at E. \& J. Gallo Winery. Thank you, Brent Sams for being my client for this project, and for being a mentor to me since the beginning of my internship at the winery. It is highly unlikely that I would have pursued a graduate degree without the advice you had given me on our long and allergy-filled days in the vineyard. Thank you, Nick Dokoozlian for approving this project and for authorizing me to be an intern for far longer than I probably should have been allowed.

Thank you to Ashley, Ava, Erick, Jessica, Jimmy, Logan, and Ty of Cohort 36 for all of the great memories. The camaraderie and bond that we all formed as a cohort is one that I will always remember. Unfortunately, our time together as graduate students was cut short, but I look forward to seeing what the future holds for us all.

Finally, I would like to acknowledge my family. I would like to thank my parents Laurie and Brian. Ultimately, I would not have embarked on this journey without both of your support. Regardless of how ridiculous my personal goals have been, you both have been there for me without question. I would finally like to thank my siblings Andrea, Matt, and Natalie for all of your encouragement and love. Overall, this project was far from an individual effort and would not have been possible without the support of everyone around me. 



\begin{abstract}
Application of Satellite Imagery for Optimal Sample Selection and Vineyard Yield Estimation

by

Brett Wong

Yield estimation has been a critical process within the field of agriculture. Traditional yield estimation methods involve field sampling, which requires either extensive knowledge of the vineyard or many random samples. New estimation techniques involving remote imagery-derived models have the potential to be more efficient than traditional methods but are relatively new to viticulture. Within this study, vineyard yield maps and satellite images were evaluated and compared for their potential use in identifying sampling locations along a single vineyard row capable of accurately representing the entire vineyard, greatly increasing sampling efficiency. The goal of this project was to determine if remote sensing techniques could assist in identifying withinfield sample locations to bridge the gap between traditional and remotely sensed yield estimation techniques.
\end{abstract}





\section{Table of Contents}

Chapter 1 - Introduction .............................................................................................. 1

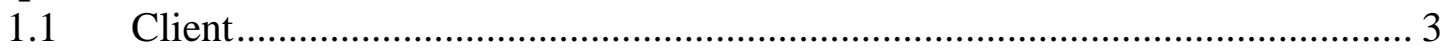

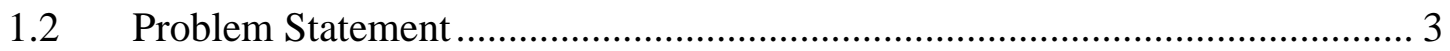

1.3 Proposed Solution ............................................................................... 3

1.3.1 Goals and Objectives ........................................................................... 4

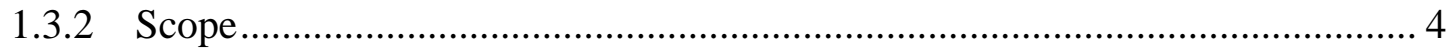

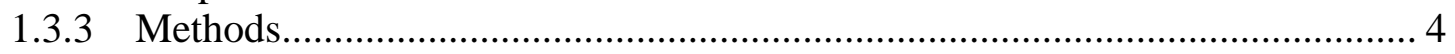

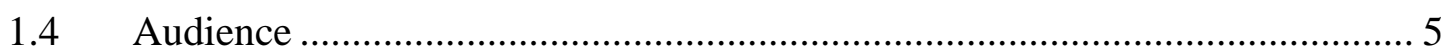

$1.5 \quad$ Overview of the Rest of this Report ......................................................... 5

Chapter 2 - Background and Literature Review ............................................................. 7

$2.1 \quad$ Precision Agriculture ........................................................................... 7

2.1.1 Understanding Vineyard Variability ..................................................... 7

2.1.2 Variable-Rate Technologies..................................................................... 7

$2.2 \quad$ Yield Estimation Techniques............................................................... 8

2.2.1 Within-Field Yield Estimation.................................................................... 8

2.2.2 Remote Imagery Estimations Using the NDVI .......................................... 9

2.2.3 Remote Imagery Estimations Using the TVI............................................... 10

2.3 Sample Site Locating Method..................................................................... 11

2.3.1 Single Vine Row Sampling Method ............................................................. 11

2.3.2 Three Pixel Sampling Method ………………….......................................... 12

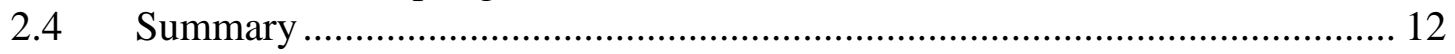

Chapter 3 - Systems Analysis and Design...................................................................... 13

$3.1 \quad$ Problem Statement .......................................................................... 13

3.2 Requirements Analysis ............................................................................... 13

3.2.1 Functional Requirements ......................................................................... 13

3.2.2 Non-Functional Requirements ............................................................... 14

$3.3 \quad$ System Design .................................................................................... 14

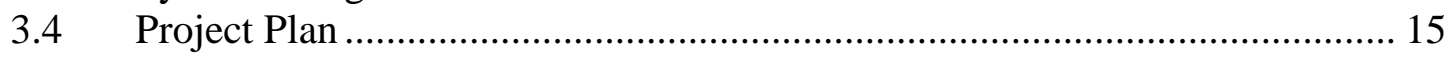

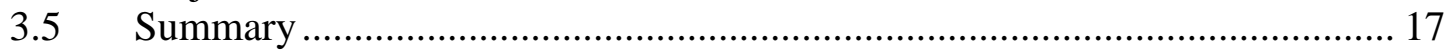

Chapter 4 - Database Design................................................................................................ 19

4.1 Conceptual Data Model .......................................................................... 19

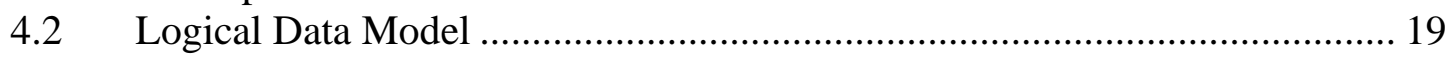

4.3 Data Sources ........................................................................................ 20

4.4 Data Cleaning and Editing ......................................................................... 21

4.4.1 Vineyard Block Boundary …………………………………………..... 21

4.4.2 Yield Map Resampling Method................................................................. 24

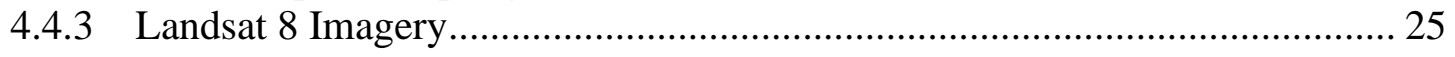

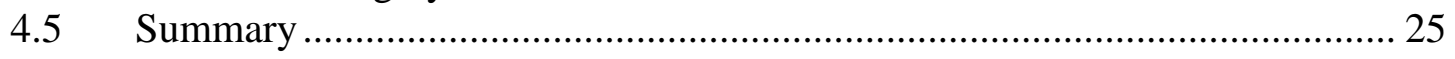

Chapter 5 - Implementation............................................................................................... 27

5.1 Yield Map and Imagery Correlation Analysis ………………………............ 27

5.1.1 Landsat Pixel Block Polygon Creation ........................................................... 27 
5.1.2 Extract Yield, NDVI, and TVI Values...................................................... 28

5.1.3 Pixel-By-Pixel Correlation Analysis........................................................... 29

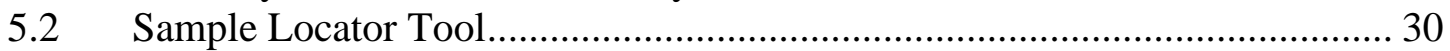

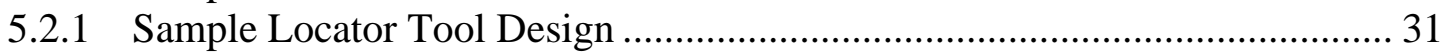

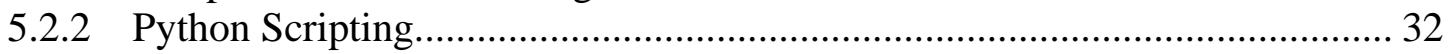

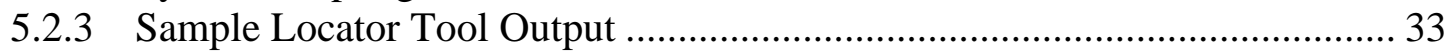

5.2.4 Random Sample Locations ……………………....................................... 34

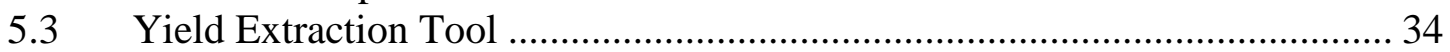

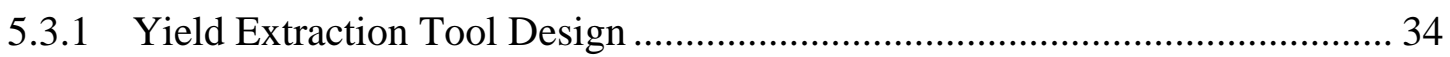

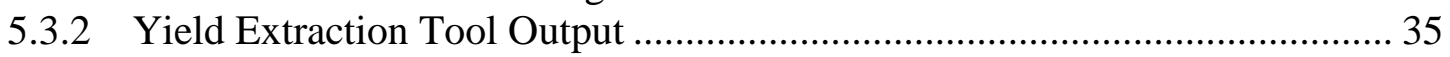

$5.4 \quad$ Yield Estimation Error Analysis ............................................................... 36

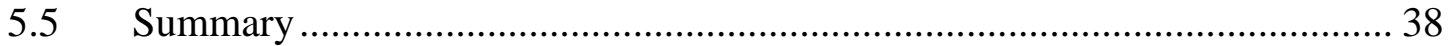

Chapter 6 - Results and Analysis...................................................................................... 39

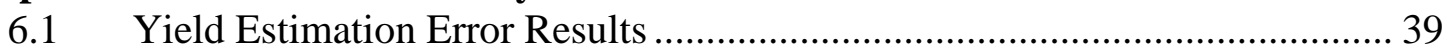

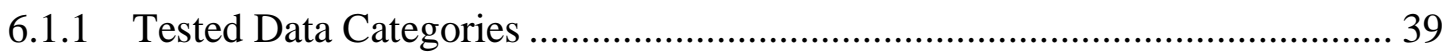

6.1.2 2017 Yield Estimation Error Results ........................................................... 40

6.1.3 2018 Yield Estimation Error Results .......................................................... 42

6.1.4 NDVI Versus TVI Yield Estimation.............................................................. 43

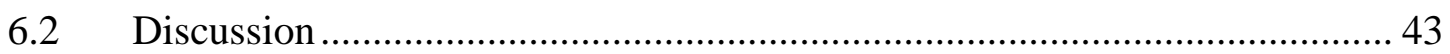

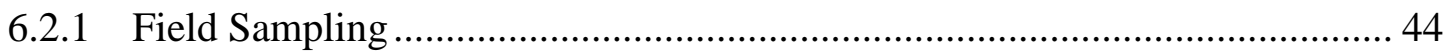

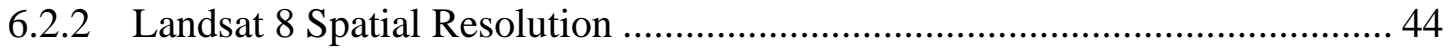

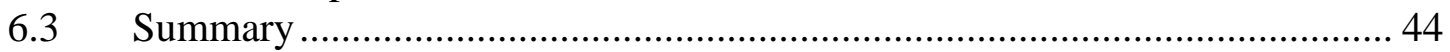

Chapter 7 - Conclusions and Future Work ................................................................ 45

7.1 Summary and Conclusion ........................................................................ 45

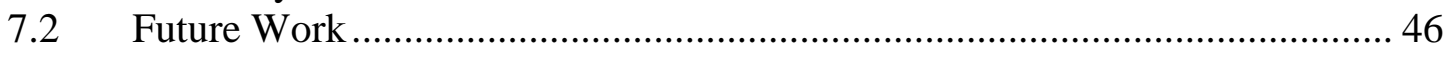

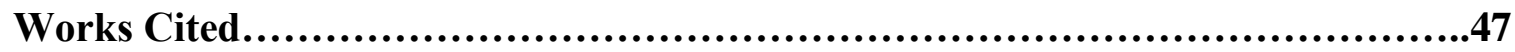

Appendix A. Sample Locator Tool ................................................................................. 49

Appendix B. Yield Extraction Tool ..................................................................................... 53 


\section{Table of Figures}

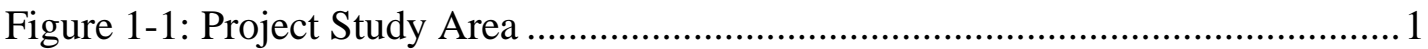

Figure 1-2: Example of a Vineyard Yield Map .................................................... 2

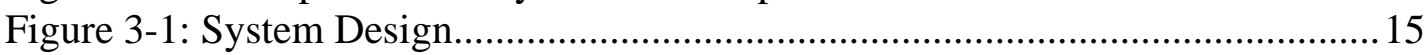

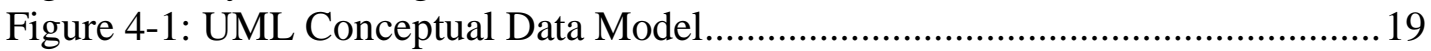

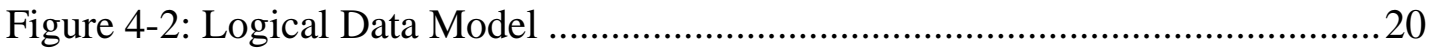

Figure 4-3: Original Boundary and Landsat Boundary Comparison........................22

Figure 4-4: Vine Angle Polyline................................................................................ 23

Figure 4-5: Vineyard Block Horizontal Row Comparison .......................................24

Figure 5-1: Landsat Pixel Block Boundary Creation.............................................28

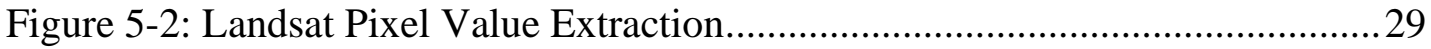

Figure 5-3: Sample Locator Tool Flowchart ............................................................ 31

Figure 5-4: Comparison of Analysis Between Horizontal and Vertical Vine Angle 32

Figure 5-5: 90 Meter Polyline and 20 Point Solution Example................................ 33

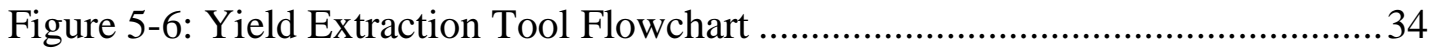

Figure 5-7: Sample Solution Extracted Yield Map Pixels and Yield Estimate .......... 36

Figure 5-8: Example of 2016 Sample Locations with 2017 Yield Map.................... 37

Figure 6-1: August 2016 TVI Solutions with Corresponding 2017 Yield Maps....... 40 



\section{List of Tables}

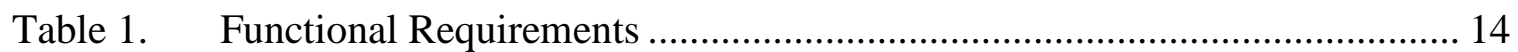

Table 2. Non-Functional Requirements ................................................................ 14

Table 3. Original Project Plan................................................................................... 16

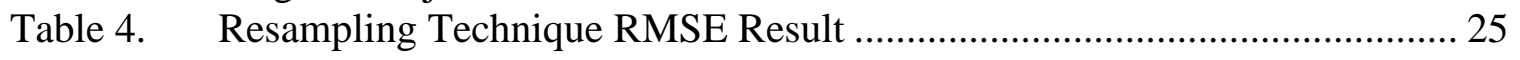

Table 5. Pixel-by-Pixel Correlation Coefficient Results Example ........................... 30

Table 6. Extracted Yield Estimations and Yield Errors for Figure 5-8 .................... 38

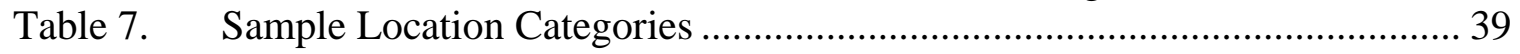

Table 8. 2017 Yield Estimation Category RMSE Ranking ................................... 41

Table 9. 2017 Yield Estimation Paired t-test Results ............................................ 41

Table 10. 2018 Yield Estimation Category RMSE Ranking …................................. 42

Table 11. 2018 Yield Estimation Paired t-test Results .............................................. 43 



\section{List of Acronyms and Definitions}

$\begin{array}{ll}\text { CCD } & \text { Canopy Chlorophyll Density } \\ \text { EVI } & \text { Enhanced Vegetation Index } \\ \text { GDAL } & \text { Geospatial Data Abstraction Library } \\ \text { GIS } & \text { Geographic Information System } \\ \text { GPS } & \text { Global Positioning System } \\ \text { IDE } & \text { Integrated Development Environment } \\ \text { LAI } & \text { Leaf Area Index } \\ \text { MAUP } & \text { Modifiable Areal Unit Problem } \\ \text { NDVI } & \text { Normalized Difference Vegetation Index } \\ \text { NIR } & \text { Near-infrared } \\ \text { PA } & \text { Precision Agriculture } \\ \text { PV } & \text { Precision Viticulture } \\ \text { RMSE } & \text { Root Mean Square Error } \\ \text { SPOT } & \text { Satellite Pour l'Observation de la Terre } \\ \text { TVI } & \text { Triangular Vegetation Index } \\ \text { UML } & \text { Unified Modeling Language } \\ \text { USGS } & \text { United States Geological Survey } \\ \text { VRDI } & \text { Variable Rate Drip Irrigation } \\ \text { VRF } & \text { Variable Rate Fertilizer } \\ \text { VRT } & \text { Variable-Rate Technologies }\end{array}$





\section{Chapter 1 - Introduction}

Yield estimation techniques have been a critical tool in the field of agriculture. As more research and new technologies progress, the efficiency and accuracy of these estimations continue to improve. Having the ability to accurately predict yield before the crop is harvested allows growers to make better planning decisions.

Though yield estimation is vital for every crop, this project concentrated on wine grapes. The study area that this project focused on is a vineyard located in California's Central Valley. The individual block boundaries of this vineyard are shown in Figure 1-1 outlined in red on an Esri imagery basemap. With over 2,200 acres of land and a large database of yield maps, this was the ideal location to test new methods of yield estimation.

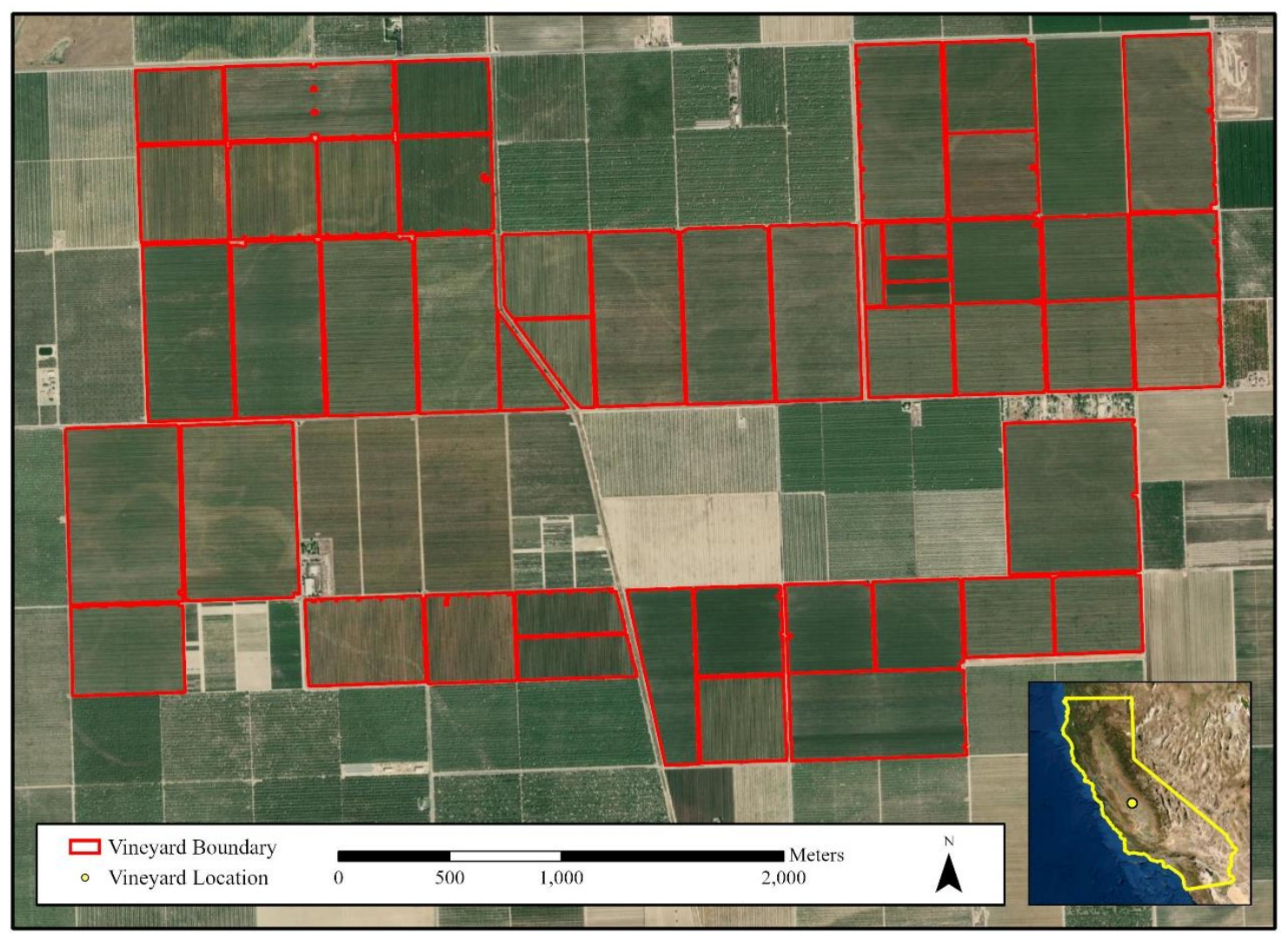

Figure 1-1: Project Study Area

Many techniques have been researched and utilized to better estimate grape yield; these techniques range from picking clusters of grapes in the vineyard to analyzing remote imagery (Dami \& Sabbatini, 2011; Sun et al., 2017). Traditional yield estimation methods involve within-field samples, which requires either an extensive knowledge of the vineyard or a large number of random samples to produce accurate results (Dami \& Sabbatini, 2011). Alternatives to traditional yield estimation techniques involve remote 
imagery-derived models that increase estimation efficiency but are relatively new to viticulture (Sun et al., 2017).

This project continued the exploration of yield estimation techniques by utilizing yield maps to test a sample locating method. Figure 1-2 below shows an example of a yield map raster of one vineyard block in the study area.

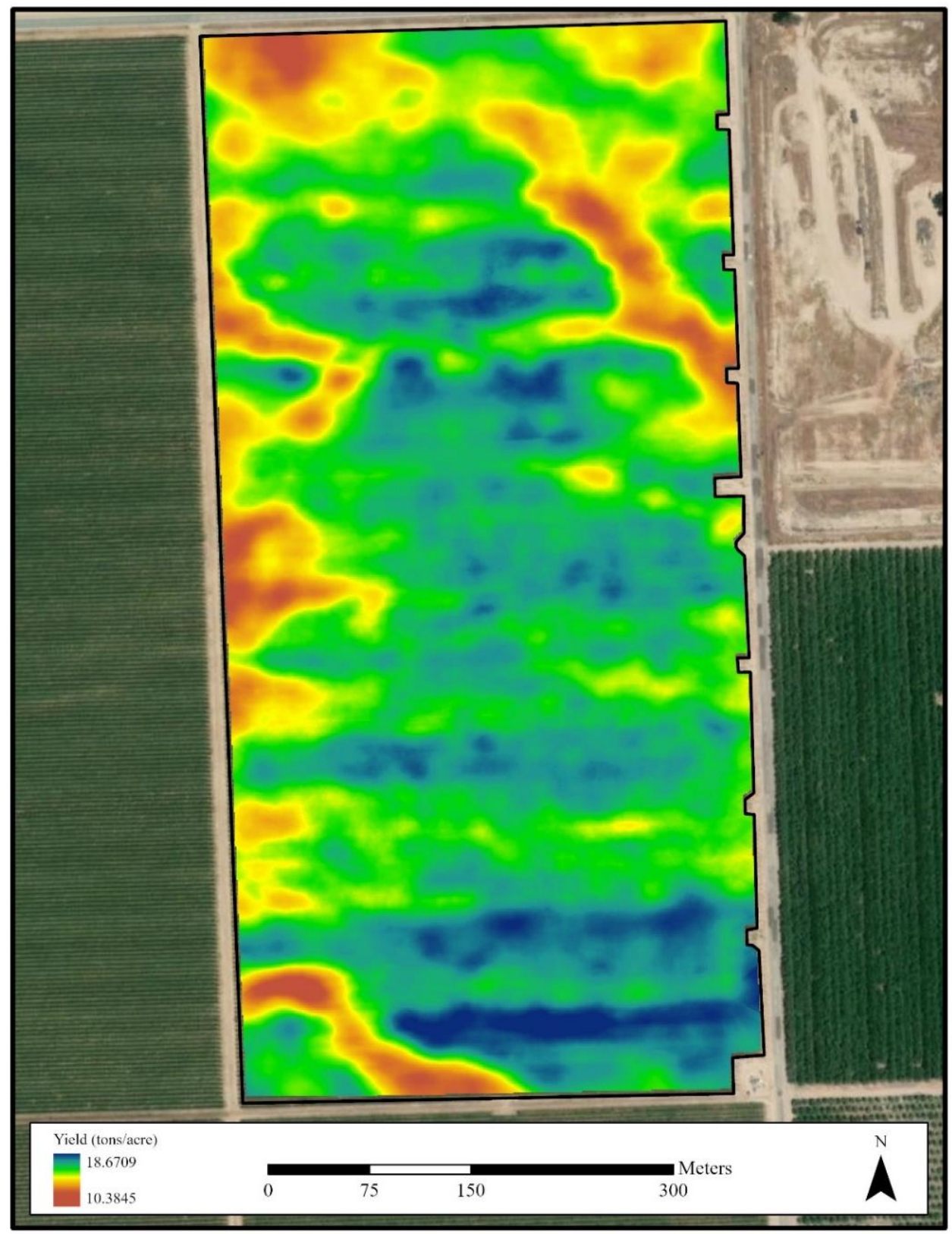

Figure 1-2: Example of a Vineyard Yield Map

These rasters are powerful because they allow for the visualization and quantification of the spatial variability of yield on a vineyard block scale. The application of these yield maps alongside remotely sensed imagery will be used to identify optimal locations for samples used in yield estimation. The effectiveness of these yield maps and remote imagery were reviewed throughout this report. 


\subsection{Client}

The client for this project is E. \& J. Gallo Winery's Viticulture Research Department. The primary point of contact at the winery was Brent Sams. He has contributed to multiple research projects that include topics in yield forecasting and mapping, precision agriculture, and vineyard cultural practices. Brent Sams is a Research Scientist in the Department of Winegrowing Research at E. \& J. Gallo Winery, focusing on the prediction of fruit composition at different spatial scales. Throughout this project, he provided guidance and industry knowledge when required.

The second point of contact for this project is Dr. Nick Dokoozlian, the Vice President of Winegrowing Research at E. \& J. Gallo Winery, and was responsible for pushing the boundaries of the development of growing practices to increase vineyard yield and improve grape quality. Before joining E. \& J. Gallo Winery, Dr. Dokoozlian was a Professor at the University of California, Davis in the Department of Viticulture and Enology.

\subsection{Problem Statement}

Acquiring accurate yield estimations is important to help growers be confident in their decisions to allocate enough resources for harvest machinery and labor, negotiate the price of their product, and determine storage space (Liu, Marden, \& Whitty, 2013). Traditional yield estimation practices worked well but required prior knowledge of the vineyard. Determining appropriate sample locations without the knowledge of spatial variability within the vineyard can lead to inaccurate results (Dami \& Sabbatini, 2011).

Yield maps have been used as an important resource to identify the spatial variability of yield within a vineyard (Bramley \& Hamilton, 2004). However, these yield maps are typically not recorded and available to growers. Remote imagery provides a viable alternative as it is available throughout the year for any area of interest. Therefore, remote imagery was processed and analyzed to determine its efficacy in identifying sampling locations that increase yield estimation accuracy.

\subsection{Proposed Solution}

Geographic information systems (GIS) and remote sensing techniques are both powerful tools that can be used in agriculture to make processes more efficient. Many studies involving remote imagery had found successful results estimating numerous vineyard metrics, such as grape quality, maturity, and yield (Meyers, Dokoozlian, Ryan, Bioni, \& Vanden Heuvel, 2020; Sun et al., 2017). Within the Viticulture Research Department at E. \& J. Gallo Winery, there was an interest to explore alternative methods of identifying optimal sampling locations to estimate yield.

The solution of this project focused on analyzing the effectiveness of applying remote imagery to identify sampling locations used to estimate yield. To assess the remote imagery, a tool was created to closely match a sample locating method that the Viticulture Research Department tested with researchers at Cornell University. This sample locating method was first tested by these two groups to estimate and summarize grape chemistry and maturity (Meyers et al., 2020). The clients at E. \& J. Gallo Winery wanted to test a similar sample locating method to determine if remote imagery can 
suggest where vineyard managers could go within the field to optimize their yield estimations and produce more accurate results.

\subsubsection{Goals and Objectives}

The goal of this project was to determine if satellite imagery could identify efficient sampling locations to estimate yield accurately. GIS and remote sensing techniques were used to validate the effectiveness of the sampling method of interest. To achieve this goal, three objectives were determined to measure the progress of this project.

The first objective set was to analyze the relationship between the yield maps and remote imagery in the form of vegetation indices. Pearson correlation analyses were used to determine which index performed best and to determine optimal time points in the season for sampling. The next objective of this project was to create tools that iterate through the yield maps and satellite imagery to output an optimal sample location. The sampling locations identified by the tools were along a single vine row intended to summarize the mean yield of its corresponding vineyard block. The final objective was to determine the effectiveness of the sample locations derived from the remote imagery. The yield estimation error between the sampling locations were compared to verify if remote imagery can be used to locate representative samples in the vineyard. Overall, the completion of these three objectives helped achieve the goal of this project.

\subsubsection{Scope}

The study area of this project focused on a vineyard owned by E. \& J. Gallo Winery located within California's Central Valley. This region was partly chosen due to the reduced likelihood of variable rate management practices. Variable-rate technologies such as variable rate drip irrigation (VRDI) could introduce deviations in yield that would be difficult to calculate (Sanchez et al., 2017). Therefore, the study area chosen was less likely to be impacted by these management techniques and should be more consistent year to year.

The Viticulture Research Department provided yield maps for 2017 and 2018. These maps were used to analyze their relationship with Landsat 8 Level-2 imagery. From the Landsat data, two vegetation indices were derived and tested. The completion of this project resulted in many tools, the main one being the Sample Locator Tool. This tool accepted yield maps or remote imagery to output sampling locations and a spreadsheet that describes their corresponding yield estimates. The methods and software used to develop these tools and analyses is described in the following section.

\subsubsection{Methods}

Many steps were implemented to achieve the goals and objectives discussed above. Before any raster analyses could occur, the remote imagery was retrieved, and vineyard block boundaries were created. The United States Geological Survey's (USGS) EarthExplorer portal was used to download Landsat 8 Level-2 images for the study area. From the satellite imagery, two vegetation indices were used to characterize the vegetative cover within the study area. Next, each yield map was resampled to match the 30 meter Landsat 8 pixel grid to allow a direct comparison between both sets of rasters. 
To complete the data preparation stage, a vineyard block boundary layer was created to delineate which pixels should be included and excluded in the raster analysis for each vineyard block.

The next step was to assess the relationships between the yield maps and vegetation indices to determine the optimal timing for sample collection. To establish this relationship, a pixel-by-pixel analysis between yield maps and vegetation indices was completed. To quantify the strength of the relationship, linear correlations were calculated between both datasets. In order to reduce the number of potential solutions, the calendar month with images most correlated to the yield maps was selected for additional analysis.

Once the required datasets were processed, the tools that determined optimal sample locations were created. The primary focus of this project was the Sample Locator Tool. The first step the tool executed was to determine the mean yield of each block. Then, a solution comprised of three contiguous pixels was identified that best represented the corresponding mean yield of the block. Finally, sampling solution locations were generated to overlap the identified three-pixel solution. A similar process using the vegetation index imagery as the tool's input was also completed.

The last step was to analyze the error between the solutions output from the yield maps and imagery. This was done using a series of paired t-tests in $\mathrm{R}$ to analyze if there were statistically significant differences amongst the solution errors. Overall, the steps listed above represent a general overview of the methods utilized to test the effectiveness of the sample locating method.

\subsection{Audience}

Overall, this project might be of interest to professionals or researchers in the field of precision agriculture. The audience that this report may be best suited for are those that are interested in the application of GIS and remote sensing for yield estimation purposes. Although many studies have been conducted using remotely sensed imagery, this project takes an alternative approach by applying yield maps and a different sample locating method to estimate yield.

\subsection{Overview of the Rest of this Report}

A brief overview will be given to summarize how the content presented in this report was organized. The next chapter will focus on previous research done for yield estimation practices. Chapter 3 will describe how the system was designed around the requirements that were given. Chapter 4 reviews the structure of the databased designed used to effectively store and retrieve data. A more detailed description of the methods is given in Chapter 5. Within Chapter 6, the results analyzed the effectiveness of yield maps and the sampling method used. To conclude this report, the final chapter will describe a few suggestions on how this research could be continued. 



\section{Chapter 2 - Background and Literature Review}

This chapter reviews the literature that helped develop the methods used in this project. First, a general overview of precision agriculture will be given in Section 2.1. Within the second section, various within-field and remotely sensed yield estimation techniques will be analyzed and compared. This section will also include an overview of vegetation indices previously used to estimate yield. Finally, Section 2.3 will describe the sample locating method that this project was influenced by.

\subsection{Precision Agriculture}

Precision agriculture (PA) practices are well established in the field of viticulture that date back to the late 1990's (Bramley \& Proffitt, 1999). The application of precision agriculture techniques to the field of viticulture have been aptly named precision viticulture (PV). Some of the goals of PV research are to develop techniques that could make the crop growing process more efficient. PV associated research covers different facets of the growing process, including the analysis of within-field vineyard variability and the development of variable-rate technologies (Arno, Martínez-Casasnovas, RibesDasi, \& Rosell, 2009).

\subsubsection{Understanding Vineyard Variability}

A key component of PV is quantifying and understanding the variability present within vineyards (Bramley \& Hamilton, 2004). Currently, vineyard maintenance such as irrigation, shoot thinning, and fertilization are typically applied evenly across a vineyard block. However, vineyard outputs vary spatially and may not follow the uniformity of the management that was applied (Arno et al., 2009). This discrepancy between uniform management and vineyard variability has led to research projects focused on key factors underpinning the variability.

There are many factors that influence the variability of yield within a vineyard. Elevation, soil type, and topography were three factors discussed by Bramley and Lamb (2003). The authors found that even though the range of elevation in their study area was only 1.2 meters, high yielding areas occurred on ridges while low yielding areas occurred in hollows. However, the authors also concluded that variations in yield had strong relationships with soil depth and available water (Bramley \& Lanyon, 2002). Overall, a better understanding of vineyard variability has led to the implementation of variable-rate technologies and the delineation of management zones (Sanchez et al., 2017; Tisseyre, Ojeda, \& Taylor, 2007).

\subsubsection{Variable-Rate Technologies}

The development of variable-rate technologies (VRT) is a key component of precision agriculture. An example of a variable-rate technology would be the variable rate drip irrigation (VRDI) system. Research by Sanchez et al. (2017) described a VRDI system that was capable of delivering customized irrigation schedules for 30 x 30 meter zones within a single vineyard block. The amount of water applied to each zone was calculated 
using NDVI imagery and weather data. The result of using this VRDI system showed that having the ability to apply varying amounts of water to different zones within the vineyard block resulted in a $17 \%$ increase in water use efficiency and a decrease in vineyard variability (Sanchez et al., 2017).

Another example of a variable-rate technology used in the field focused on the application of fertilizer. Variable rate fertilization (VRF) is a tool that allows differing amounts of fertilizer to be applied to the crop being managed. According to Schumann (2010), VRF is made possible by the interaction between an accurate Global Positioning System (GPS), GIS, remote sensing, actuators, and sensors that measure crop properties. The benefit of VRF is it allows the grower to apply a varying amount of fertilizer to the crop depending on many factors, such as canopy vigor, soil type, and water content. Overall, the use of a VRF system results in a more efficient application of fertilizer which leads to less waste and reduced costs.

Overall, it will be vital to continue the research and development of precision agriculture techniques that make the growing cycle more efficient. Increasing resource use efficiency can better protect growers from resource shortages and decrease maintenance costs.

\subsection{Yield Estimation Techniques}

Many yield estimation techniques have been researched and implemented to estimate vineyard yield, which can be categorized as either within-field (Dami \& Sabbatini, 2011) or remotely sensed (Hall \& Wilson, 2013; Sun et al., 2017) estimations. Examples of both within-field and remotely sensed yield estimation techniques will be reviewed in the following sections.

\subsubsection{Within-Field Yield Estimation}

The more traditional methods used to estimate yield are derived from samples gathered in the field. These methods are generally more trusted over remotely sensed estimations because physical clusters of grapes are sampled and recorded. However, manually collecting samples in the vineyard can be time consuming and costly.

Although specific yield estimation methods differ amongst growers, most follow a general calculation. The yield estimation (in tons per acre) is calculated as

$$
\mathrm{YE}=\frac{(\mathrm{ANV} \times \mathrm{NC} \times \mathrm{CW})}{2000}
$$

where ANV is the actual number of vines per acre, $\mathrm{NC}$ is the number of clusters per vine, and CW is the cluster weight in pounds (Dami \& Sabbatini, 2011).

The simplest parameter to calculate is the actual number of vines per acre. This value can be determined by measuring the vine spacing and row spacing of the vineyard block being estimated (Komm \& Moyer, 2015). A key factor when calculating this value is the number of vines that do not produce grapes. If nonproducing vines are not considered, the estimated yield will be higher than the actual yield. The process that deviates most between growers is measuring grape clusters per vine. Dami and Sabbatini (2011) suggested using a methodical process by sampling vines that are evenly spaced apart. The 
final parameter measured is the cluster weight, which can be calculated multiple ways. One method suggests removing all the clusters from a single vine and dividing their total weight by the number of clusters picked (Komm \& Moyer, 2015). With all three parameters measured, a yield estimation can be calculated to help growers make more informed planning decisions.

Overall, the key decision of this process is choosing the locations of the vines to sample. The accuracy of yield estimates can vary based on how closely the sampled vines resemble the entire block. This could lead to an overestimation or underestimation depending on the vigor of the sampled vines relative to the entire yield of the block. One approach of eliminating the possibility of choosing unrepresentative vines is by using remote imagery to directly estimate yield or to identify sampling locations.

\subsubsection{Remote Imagery Estimations Using the NDVI}

Remote imagery has become an increasingly important source of data in precision agriculture research (Arno et al., 2009). Vegetation indices have been used to help characterize and understand the variability of vegetative cover throughout the vineyard (Sun et al., 2017). These vegetation indices can be derived to help accomplish tasks such as determining the amount of grape varieties in a vineyard and mapping relative vigor variability within a block (Hall, Lamb, Holzapfel, \& Louis, 2002). As a result, many studies involving remote imagery have been developed to determine how well vegetation indices perform to estimate yield.

One study that focused on the relationship between regional grape yield and remote imagery was described by Cunha, Marcal, and Silva (2010). Their approach was to develop a wine yield forecast system to model the relationship between remote imagery and regional vineyard yield. The source of remote imagery used in this study was from the Satellite Pour l'Observation de la Terre's (SPOT) Vegetation sensor, which has a spatial resolution of one kilometer. From the Vegetation sensor, a time series dataset of Normalized Difference Vegetation Index (NDVI) imagery was used to characterize the vegetative cover of the four regions in Portugal. To develop the model, a linear regression was fitted to determine the relationship between the regional vineyard yields and their overlapping NDVI imagery. The results of this model suggest that $77-88 \%$ of the regional vineyard yield was explained by the model using April NDVI imagery from previous years. These results are promising, as they show that remote imagery can assist in estimating yield before the harvest season begins.

Sun et al. (2017) found that remote imagery could be used to better understand and estimate yield. The relationship between vineyard yield and NDVI imagery was summarized to help build a model used to estimate yield. A key aspect that differentiated the analyses described within Sun et al. (2017) from the previous yield estimation research was its implementation of vineyard yield maps, similar to those described in Chapter 1 . These yield maps were resampled to 30 meters to compare directly to the Landsat NDVI imagery. A correlation analysis comparing resampled yield maps and monthly NDVI imagery to determine when the peak correlation occurred between both datasets. Within this research, the yield maps were used to gain a better understanding of the relationship between yield and remote imagery.

The NDVI was first documented by Rouse, Haas, Schell, and Deering (1974), which was developed to measure forage vegetation for cattle and crop production in the Great 
Plains. Since its creation, this index has been widely used across many fields of study including agriculture and environmental sciences (Li, Yan, \& Fan, 2005; Sun et al., 2017). The normalized difference vegetation index is calculated as

$$
\mathrm{NDVI}=\frac{\left(R_{\mathrm{NIR}}-R_{\mathrm{Red}}\right)}{\left(R_{\mathrm{NIR}}+R_{\mathrm{Red}}\right)}
$$

where $R_{\text {NIR }}$ is the spectral reflectance of the near-infrared (NIR) band and $R_{\text {Red }}$ is the spectral reflectance of the red band (Rouse et al., 1974). The NDVI focuses on the difference in spectral reflectance values of the red and near-infrared (NIR) bands. Typically, healthy green vegetation reflects most NIR radiation and absorbs red radiation, resulting in a high NDVI value (Gamon et al., 1995). The result of this relationship suggests that healthy green vegetation will have higher NDVI values while less green vegetation will have lower NDVI values. As shown in Cunha et al. (2010) and Sun et al. (2017) this vegetation index has aided in the development of models used to estimate yield.

\subsubsection{Remote Imagery Estimations Using the TVI}

Another vegetation index that has received attention in precision agriculture research is the triangular vegetation index (TVI). The TVI was analyzed by Hall and Wilson (2013) in a study that compared the correlation between yield, grape composition, and multiple vegetation indices. The performance of many vegetations indices were analyzed including the TVI, NDVI, and enhanced vegetation index (EVI). To determine which index correlated best with yield and grape composition, correlation analyses were done between the imagery and samples taken in the vineyard. Hall and Wilson (2013) found that the vegetation index that had the highest amount of significant correlations with yield and grape composition was the TVI. With these results it was suggested that the TVI had a "potential superior ability to more accurately forecast spatial patterns of vineyard fruit quality and yield" (Hall \& Wilson, 2013, p. 1790).

The TVI was developed by Broge and Leblanc (2000) as another source of comparison to determine which vegetation index best predicted leaf area index (LAI) and canopy chlorophyll density (CCD). The triangular vegetation index is calculated as

$$
\mathrm{TVI}=0.5\left(120\left(R_{\mathrm{NIR}}-R_{\mathrm{Green}}\right)-200\left(R_{\mathrm{Red}}-R_{\mathrm{Green}}\right)\right) \quad \text { Eq. 2-3 }
$$

where $R_{\text {NIR }}, R_{\text {Red }}$, and $R_{\text {Green }}$ represent the spectral reflectance of the NIR, red, and green bands respectively (Broge \& Leblanc, 2000). The coefficient of 120 in Equation 2-3 represents the difference in the wavelengths between the red band $(670 \mathrm{~nm})$ and the green band $(550 \mathrm{~nm})$, where the coefficient of 200 represents the difference in the wavelengths between the NIR band $(750 \mathrm{~nm})$ and the green band.

One of the main differences between TVI and NDVI is the use of the green reflectance band. "The index is calculated as the area of the triangle defined by the green peak, the chlorophyll absorption minimum, and the NIR shoulder in spectral space." (Broge \& Leblanc, 2000, p. 162). Similar to the NDVI, the TVI measures vegetation based on the difference in spectral reflectance in the NIR and red bands. Healthier green vegetation will result in a larger TVI value as spectral reflectance will be high in the NIR 
band and low in the red band. Although the TVI was not the best performing VI to predict LAI or CCD, this index sparked interest in other studies and applications. Overall, there have been many studies that have shown successful results of using the NDVI and TVI to help estimate yield (Cunha et al., 2010; Hall \& Wilson, 2013; Sun et al., 2017).

\subsection{Sample Site Locating Method}

The sampling method implemented in this project is similar to the one that was developed by researchers at Cornell University and later in collaboration with E. \& J. Gallo Winery. The development of this sampling location method is broken down into two sections. The first section focused on a study that assessed the performance of sample locations that were taken from a single vine row within a vineyard block. The second section reviews how this single vine row sampling method was used to estimate the grape maturity of $\mathrm{E}$. $\& \mathrm{~J}$. Gallo vineyards.

\subsubsection{Single Vine Row Sampling Method}

In the search to develop a more efficient sampling method, Meyers and Vanden Heuvel (2014) researched the effectiveness of single vine row sampling. The goal was to determine if samples taken from an individual vine row could summarize the characteristics of its entire block. Compacting the samples from randomly dispersed locations to a single vine row would make the process more efficient.

To test this sampling method, Meyers and Vanden Heuvel (2014) used high resolution NDVI imagery that was split into six separate analysis zones. For each zone, many sample sizes were tested for the three different sampling categories used: randomly generated, globally optimized, and single vine row. The random samples were created by choosing random pixels within the zone, the globally optimized solutions could choose from any pixels in the zone, and the single row method was limited to pixels within an individual vine. These sample files were used to extract the pixel values from the NDVI imagery of each zone to represent the outcome of each sample.

To determine the effectiveness of the samples generated from the three sampling categories, a function was used to compare the difference between the NDVI of the whole zone to the NDVI of the zone's corresponding samples. The study found that the globally optimized and single row solutions required a smaller sample size (image pixels) to summarize the variation in NDVI of the entire zone (Meyers \& Vanden Heuvel, 2014). One drawback of the globally optimized location was that the selected pixels were dispersed similarly to the random samples. Physically collecting these samples in the field would require more travel time than the single vine row solution. Overall, the single row method was the best option because the pixels were representative of the zone and required less traveling between samples.

The concept that representative sampling locations could be derived from a single vine row was appealing to the Viticulture Research Department. This interest resulted in the collaboration between both parties, which will be described in greater detail below. 


\subsubsection{Three Pixel Sampling Method}

In collaboration with E. \& J. Gallo Winery, Meyers and Vanden Heuvel tested the single row sample locations on a larger scale. The objective of this research was to test if samples taken from a single vine row could accurately estimate grape maturity and quality within a vineyard block. If the samples derived from this technique accurately described the maturity of a given block, then it would make the maturity sampling process more efficient.

To analyze the effectiveness of this technique, three sampling methods were tested: random, point quadrant, and single vine row. The random sampling was done by generating 20 random sample locations for each block, the point quadrant was identified by selecting one sampling location within each quadrant of the block, and the single row method focused on sampling an individual vine row (Meyers et al., 2020).

The main focus of this study was the single vine row solutions. The first step taken to identify the location of the single row solutions was to prepare the required data inputs. The first input was a vineyard block boundary that contained the vine angle for each polygon and the second input was the NDVI dataset derived from Landsat 7 imagery. Together, both inputs were run through an algorithm to locate optimal single row solutions.

The algorithm created by Meyers et al. (2020) iterated through each vineyard boundary, analyzed every pixel within the block, and calculated its corresponding three quantile NDVI mean that represented the left tail, center, and right tail. Every consecutive three pixel solution was analyzed to determine which three pixels best matched the population three quantile mean. Once the random, point quadrant, and single vine row solutions were created, samples were collected in the field for the designated locations. Meyers et al. (2020) found that the samples from the single vine row more accurately described grape maturity and chemistry than the random and quartile sampling methods.

The successful results of the single vine row sampling locations interested the Viticulture Research Department to explore other applications of this sampling technique. This interest led to the creation of the methods described in this project, which tested the validity of the single vine row samples to estimate vineyard yield.

\subsection{Summary}

The studies reviewed in this chapter make up a small subset of the research that has been done in the field of PA. According to this research, it is clear that it will be important to continue making agricultural practices more efficient. The primary focus of this project is to explore an alternative approach to estimate yield. There are two main categories of yield estimation used in the vineyard: within-field and remotely sensed techniques. This project seeks to test a hybrid solution that analyzes imagery to output field sampling locations to estimate yield. The system design and planning used to develop the methods required to test the accuracy of single vine row solutions is documented in the following chapter. 


\section{Chapter 3 - Systems Analysis and Design}

This chapter describes the system analysis and design that was established to complete the project in an efficient manner. In Section 3.1, the problem the client wanted to address is revisited. The following section describes the requirements that were used to determine how successful the project was. Section 3.3 gives a visual representation of the system design used to address the requirements. In the final section, the project plan was outlined and defined in more detail. Overall, these four aspects make up this project's system analysis and design.

\subsection{Problem Statement}

Yield Estimation is a vital step in modern agricultural processes. Traditionally, yield estimation requires prior knowledge of the vineyard to determine where representative samples should be taken. If a group of representative vines is unknown, one of the best options is to sample at a set vine spacing (Dami \& Sabbatini, 2011). However, this method is less efficient as it requires the sampler to walk throughout a larger portion of the field to obtain an accurate estimation.

Yield maps have been a new source of data to help better understand the spatial variability of yield within a vineyard. These yield maps from previous harvests have been used to delineate vineyard management zones and create yield estimation models (Sun et al., 2017; Tisseyre et al., 2007). However, yield maps are a resource few wineries have access to. In this study, remote imagery has been proposed as an alternative method of quantifying variability within the vineyard.

\subsection{Requirements Analysis}

The requirements established by the client's goal can be categorized as functional or nonfunctional requirements. Functional requirements can be described as the essential behaviors the system should perform. In contrast, non-functional requirements are the constraints that determine how the system should operate. An overview of both types of requirements for this project will be given in the following sections.

\subsubsection{Functional Requirements}

The functional requirements of this project were established to ensure that the tools and data analysis met the client's expectations. The first requirement was to develop a tool that located optimal sampling solutions similar to the methods documented in Meyers et al. (2020). This tool's primary functions were to iterate through every yield map in the study area, determine the yield of the block, and find the three-pixel solution that matched the mean of each block. Both NDVI and TVI imagery were derived from the Landsat 8 rasters to load into this tool. The final requirement was to statistically analyze how well the sample locations derived from the tool estimated yield. A breakdown of the functional requirements can be seen in Table 1. 
Table 1. Functional Requirements

\begin{tabular}{|l|l|}
\hline Requirement & Description \\
\hline Locate Sample & Use NDVI and TVI imagery to locate sample locations using \\
Solutions & methods similar to those documented in Meyers et al. (2020) \\
Sample Fitness & $\begin{array}{l}\text { Run statistical analyses to determine which data source } \\
\text { provides the most accurate yield estimations }\end{array}$ \\
\hline
\end{tabular}

\subsubsection{Non-Functional Requirements}

The two non-functional requirements came in the form of preferred software and are shown in Table 2. The first request was to ensure that all tools were compatible with ArcMap. The Viticulture Research Department currently use ArcMap as their primary GIS software, so the delivered tools were created to work within this program. The final non-functional requirement was to use Python as the scripting language. The benefit of using Python is that it gives access to the ArcPy library, which is useful for automating geoprocessing tools. Overall, these functional and non-functional requirements were used to set the guidelines needed to address the client's goal.

\section{Table 2. Non-Functional Requirements}

\begin{tabular}{|l|l|}
\hline Requirement & Description \\
\hline ArcMap & $\begin{array}{l}\text { ArcMap was required to access to the geoprocessing tools } \\
\text { required to locate samples } \\
\text { Python was the used to automate the geoprocessing tools } \\
\text { Peeded to locate samples. }\end{array}$ \\
\hline
\end{tabular}

\subsection{System Design}

The system design established the general workflow used to address the Viticulture Research Department's goal. To determine the viability of the sample locating method of interest, three tools were created. Figure 3-1 illustrates the organization of the system design and how these tools worked together to achieve this goal. 


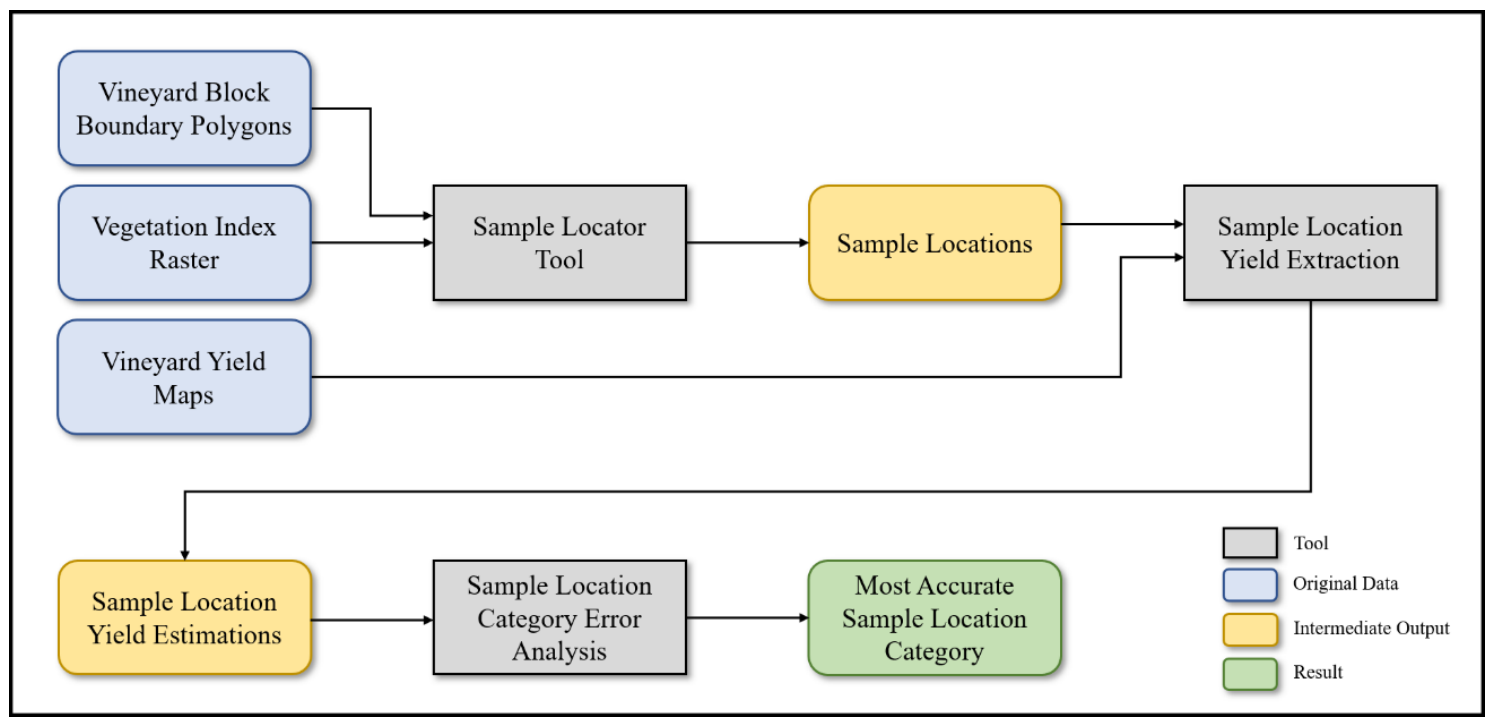

Figure 3-1: System Design

The primary tool created for this project was the sample locator script that accepted either satellite imagery or yield maps to determine where the grapes should be sampled. Although this tool located sampling solutions in the desired method, the accuracy of their estimations needed to be tested. Therefore, the second tool was created to iterate through every sample location to extract the estimated yield values from its corresponding yield map. After the estimated yield was extracted, the error was defined as the difference between the yield estimate and the true yield of the vineyard block. The final tool analyzed these errors to determine if the sample locations derived from satellite imagery were accurate.

\subsection{Project Plan}

The project plan was divided into five separate phases: plan, design, develop, test, and deploy. The purpose of these phases was to break the project into manageable tasks that were used to determine the time required and the progress made for each task. A breakdown of the phases and tasks that make up the original project plan can be seen in Table 3. 
Table 3. Original Project Plan

\begin{tabular}{|lllll|}
\hline Phase & Description & Hours & Start Date & End Date \\
\hline $\mathbf{1}$ & Plan & & & \\
\hline 1.1 & Requirement Analysis & 15 & Dec - 2 & Dec - 5 \\
1.2 & Literature Review & 150 & Dec - 2 & May - 20 \\
1.3 & Plan Schedule & 10 & Jan - 6 & Jan - 10 \\
1.4 & Data Collection and Creation & 10 & Jan - 12 & Jan - 17 \\
1.5 & Clean Data & 30 & Feb - 1 & Feb - 12 \\
1.6 & Chapters 1 and 2 Documentation & 100 & Feb - 17 & Apr - 1 \\
\hline $\mathbf{2}$ & Design & & & \\
\hline 2.1 & Pilot Test (Sample Locator Tool) & 30 & Mar - 10 & Mar - 30 \\
2.2 & Design Database & 30 & Mar - 10 & Mar - 30 \\
2.3 & Chapters 3 and 4 Documentation & 100 & Apr - 3 & May - 30 \\
\hline $\mathbf{3}$ & Develop & & & \\
\hline 3.1 & Develop Sample Locator Tool & 100 & Apr - 4 & Apr - 30 \\
3.2 & Develop Data Extraction Tools & 40 & May - 2 & May - 12 \\
3.3 & Develop Data Analysis Tools & 20 & May - 14 & May - 18 \\
3.4 & Chapter 5 Documentation & 60 & May - 20 & May - 31 \\
\hline $\mathbf{4}$ & Test & & & \\
\hline 4.1 & Test Output of Sample Locator Tools & 20 & Jun - 1 & Jun - 5 \\
4.2 & Test Data Extraction and Analysis Tools & 20 & Jun - 7 & Jun - 11 \\
4.3 & Report and Fix Bugs & 30 & Jun - 13 & Jun - 18 \\
4.4 & Analyze Data & 40 & Jun - 20 & Jun - 28 \\
4.5 & Chapters 6 and 7 Documentation & 100 & Jul - 1 & Jul - 18 \\
\hline $\mathbf{5}$ & Deploy & & & \\
\hline 5.1 & Review Results with Clients and Advisor & 5 & Jul - 20 & Jul - 20 \\
5.2 & Finalize Report & 40 & Jul - 22 & Jul - 29 \\
5.3 & Deliver Report and Tools to Clients & 5 & Jul - 31 & Jul - 31 \\
\hline & & & \\
\hline
\end{tabular}

The first section of the project was the planning phase where a majority of the project tasks were organized. Another key component of this section was the literature review, which helped summarize the current yield estimation practices and research the methods used in this project. With a better understanding of the overall plan and literature, the required imagery was downloaded, and the vineyard boundary file was created.

After the plan was set, the next step was to design the Sample Locator Tool and database structure. These two tasks were worked on concurrently due to their relationship. The database needed to be structured in an organized manner that made logical sense. The two goals of the pilot test were to evaluate the initial methods and determine if the database was structured appropriately. In order to move onto the next phase, edits were made to the database structure and pilot test until the outcome was acceptable.

The third phase of the project dealt with the development of the three primary tools listed in Table 3. After the system design (Figure 3-1) was created, the function and 
relationship between these three tools became clear. The Sample Locator Tool analyzed yield maps or remote imagery to identify where the single vine row samples were located. The Yield Extraction Tool focused on determining the yield estimations for each of the sampling locations that were output from the previous tool. Finally, the error analysis tool determined the performance of the sample solutions derived from remote imagery and Sample Locator Tool.

Once the tools were completed, the next phase was to test their results. To determine that the outputs of each tool were correct, manual quality control checks were done on a random subset. If any sample location was outside of the block boundary or in an orientation that did not match the vine direction, the error was later fixed.

The last phase of this process was to deploy the project. First, the results were reviewed with all of the stakeholders to ensure that every aspect of the project was sound. Afterwards, the report was finalized making the recommended changes. Finally, the report and tools were delivered to the client.

Although the project plan was followed as close as possible, there were some unexpected occurrences that altered the plan. The largest change came within the development phase. The methods of the Sample Location Tool were straightforward, but the data extraction and analysis tools required many iterations. As the project progressed, different data extraction and analysis methods were explored. This exploration resulted in a more robust methodology but required more time than was initially planned. Despite this loss in time, the output Excel files of the edited tools were more concise, making the analysis more efficient. Overall, the project plan (Table 3) was a critical reference resource to determine the progress made throughout the project.

\subsection{Summary}

Within this chapter, the requirements, system design, and project plan were reviewed. The primary goal of developing these resources was to ensure that the project addressed the Viticulture Research Department's inquiry within the given time frame. After the project plan was established, the next step was to gather the required data and create the database. 



\section{Chapter 4 - Database Design}

This chapter reviews the database design, data gathering, and data processing completed for this project. The purpose of these three processes was to ensure that the required datasets were organized and prepared in a way that could address the client's goal. Creating a database structure for commercial use was beyond the scope of this project, however a sound database would greatly benefit the efficiency of the tasks required. Without a well-structured database and properly processed data, it is increasingly difficult to produce meaningful results. Therefore, it was important to understand the intended interactions between datasets and their final format.

\subsection{Conceptual Data Model}

Before any GIS processes or analyses could occur, a conceptual data model was created. The main function of this data model was to determine the relationships between entities within the database. Figure 4-1 displays the conceptual data model as a Unified Modeling Language (UML) class diagram, which is presented below.

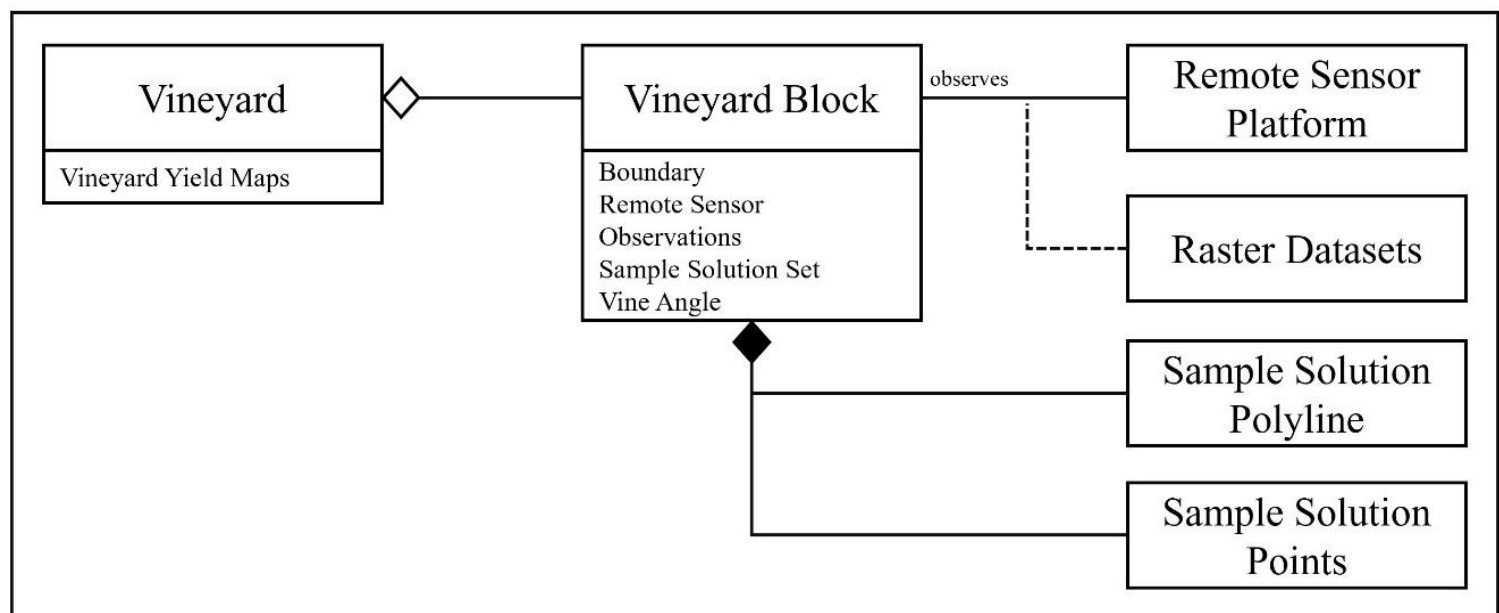

Figure 4-1: UML Conceptual Data Model

The study area of this project was a vineyard located in California's Central Valley. From the vineyard, the block boundary polygons and yield maps were created by the Viticulture Research Department. Landsat 8 was used as the remote sensor platform to observe the vineyard blocks of the study area. Both satellite imagery and yield maps were then used to output sample solution polylines and points for each corresponding vineyard block. Overall, the relationships shown in this conceptual data model solidified the workflow and interactions between each entity.

\subsection{Logical Data Model}

The logical data model was developed to expand on the framework that was built by the conceptual data model. Its primary function was to specify the attributes of the entities within the conceptual model. These attributes are shown in the logical data model (Figure 4-2). 


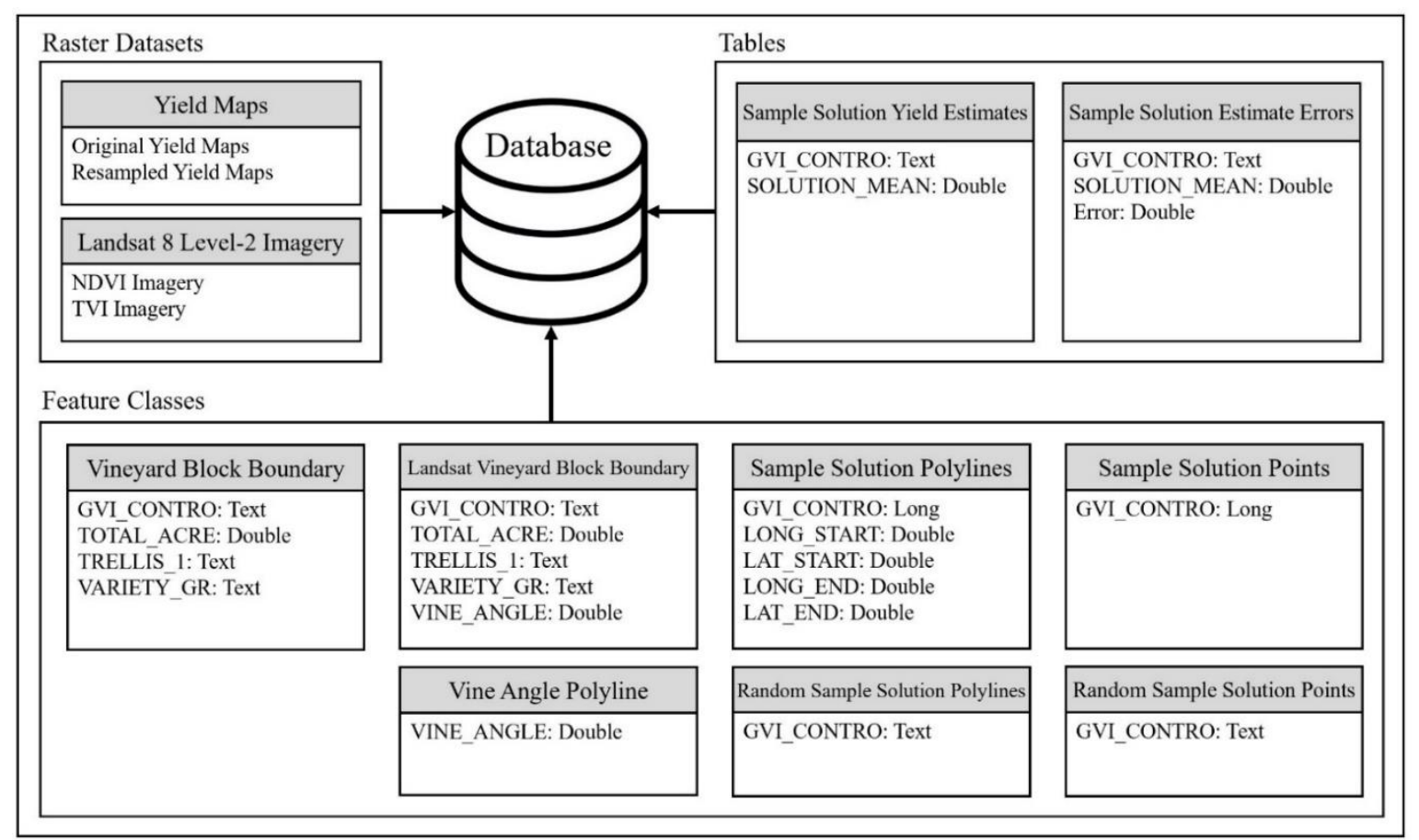

Figure 4-2: Logical Data Model

There are many feature classes, raster datasets, and tables contained within the database. The feature classes make up all of the block boundary and sample solution files required for analysis. An important attribute present in most of the feature classes is the vineyard block identifier, which is labeled as GVI_CONTRO. This identifier was the key to analyzing all of the vineyard blocks individually. The raster datasets include the yield maps and remote imagery, which were used as the inputs for the sample locator tools. Finally, the tables were used to summarize the yield estimates and errors derived from the sample solutions.

One of the notable database design choices was to forego the use of an Esri file geodatabase. The reason for the omission of a file geodatabase was the use of the Geospatial Data Abstraction Library (GDAL). This is a Python library that was utilized in the Sample Locator Tool to read and analyze the remote imagery. One drawback of using the GDAL is its inability to read raster datasets within Esri file geodatabases. Therefore, a folder structure was used to store the required datasets. Together, the conceptual and logical data models were used to help create the structure of the database used to organize the necessary datasets.

\subsection{Data Sources}

The two main sources of data were E. \& J. Gallo's Viticulture Research Department and the United States Geological Survey (USGS). To test the sampling method, the clients provided a vineyard block boundary shapefile and yield maps from multiple years. The purpose of the boundary file was to delineate the individual blocks that made up the entire vineyard. This boundary file contained key metadata, such as the block identifier 
and grape variety. Yield maps from 2017 and 2018 were provided by the client that summarized the spatial variation of yield in each block. These yield maps were used to determine what the estimated yield would be at any sample location output by the tools.

After the necessary datasets were received from the Viticulture Research Department, the next step was to download the imagery. Imagery required for analysis was readily available on the USGS Earth Explorer portal. For the study area, Landsat 8 Level-2 imagery was downloaded from March to October for each year the yield maps were available. The Landsat satellite program was chosen as the data source because it has played an important role in viticulture research at E. \& J. Gallo Winery and is freely available to the public. With all the required data gathered, the next step was to clean and load these datasets.

\subsection{Data Cleaning and Editing}

Before the individual datasets were ready for analysis, each set required cleaning and editing. The goal of these edits was to prepare these datasets in a workable format for analysis. The following sections give a detailed overview of the edits made to the vineyard boundary polygon, yield maps, and Landsat 8 imagery.

\subsubsection{Vineyard Block Boundary}

The first dataset that required editing was the vineyard block boundary. The first edit focused on recreating the boundaries while the second edit was to extract the orientation of the vine rows for each block.

The vineyard boundary file was used to clip the 30 meter pixels from the Landsat imagery that belonged to each block. In its original form, the vineyard boundaries extracted "mixed pixels", which were pixels that contain any objects that are not vineyard canopy. In this application, dirt paths, bodies of water, and roads are the main causes of these pixels. For this analysis, a new boundary file was created manually to ensure that these pixels were removed from the analysis. Figure 4-3 shows an example of the difference between using the original boundary and the new Landsat block boundary to extract the pixels for the block. In this example, the original boundary includes mixed pixels on every side, while the Landsat boundary clips those pixels out. A new Landsat boundary was created for every vineyard block in the study area to ensure that mixed pixels were not included in the analysis. 


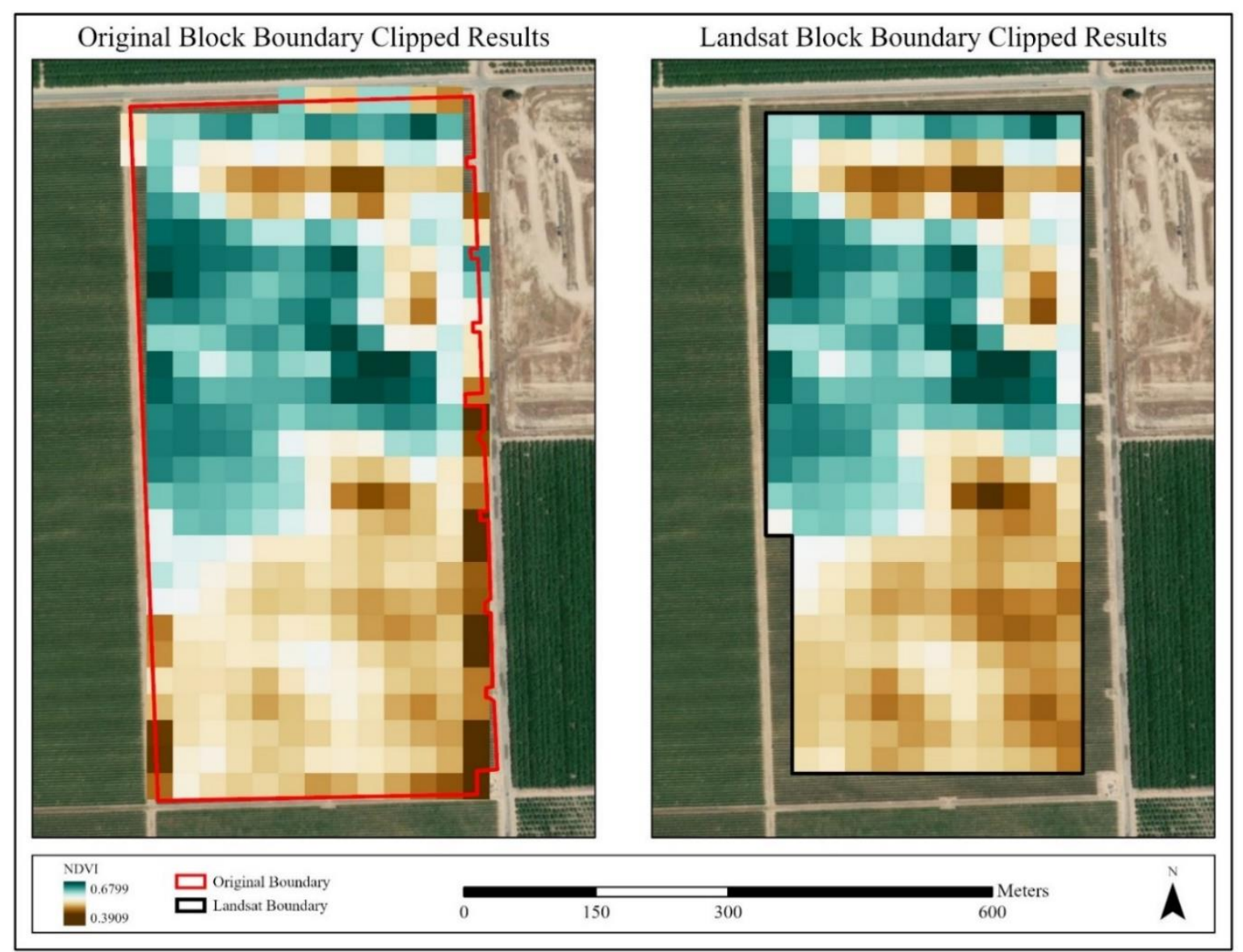

Figure 4-3: Original Boundary and Landsat Boundary Comparison

Following the completion of the new Landsat boundaries, the final edit was to determine the vine row angle of every block. The orientation of the vines was a key attribute, which established how the imagery would be analyzed and the direction of the sample solution locations. To find the vine angle of every block, a separate polyline feature was manually digitized along the vine rows of each block. Figure 4-4 displays one block with its corresponding vine angle polyline in green. In this example, the vine angle of this block is 88.90 degrees. 


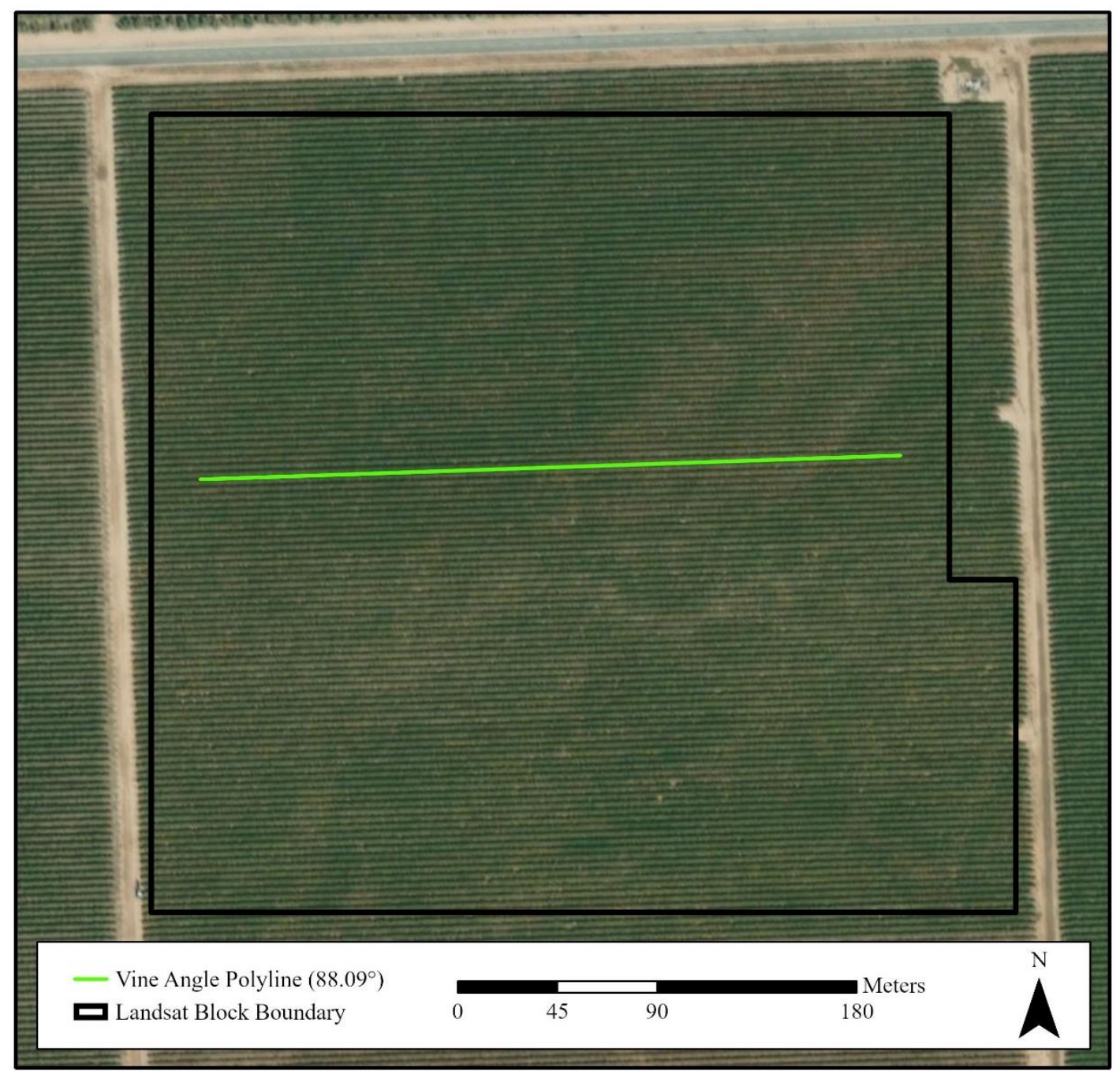

\section{Figure 4-4: Vine Angle Polyline}

After all of the vine angle polylines were created, their corresponding angles were calculated by the Linear Directional Mean geoprocessing tool in ArcMap. These angles were then transferred to the Landsat boundary polygon with the Spatial Join geoprocessing tool. These two changes resulted in a new boundary layer that clips the appropriate imagery pixels and notifies the Sample Locator Tool of each block's vine angle.

One issue with this Landsat boundary file is that the process of creating this dataset is time consuming. Although a similar process was completed for a previous E. \& J. Gallo research project, it is unlikely that a commercial dataset could be created for all of the properties that are owned or contracted out by the winery. However, creating a similar boundary file would be manageable for a subset of vineyards the winery is particularly interested in. 


\subsubsection{Yield Map Resampling Method}

The second set of data that needed to be prepared were the yield maps. In their original format, these rasters had a resolution of one meter. To compare the yield maps directly to the Landsat imagery, they had to be resampled to match the Landsat 8 resolution of 30 meters. An issue that was considered while resampling the yield maps was the modifiable areal unit problem (MAUP). One aspect of the MAUP is the scale effect which is defined as the, "variation in results that may be obtained when the same areal data are combined into sets of increasingly larger areal units of analysis" (Openshaw \& Taylor, 1979, p. 128). In this application, the scale effect is present when increasing the areal unit of the imagery from one meter to 30 meters. Although the scale effect was an issue when resampling the yield maps, tests were done to ensure the correct technique was used.

The Resample geoprocessing tool within ArcMap has three techniques: nearest, bilinear, and cubic. To determine which technique was the best fit for this dataset, a single vineyard block was chosen to test their results. This block was separated into 26 horizontal rows for comparison. These horizontal zones were compared row by row to measure the change in mean yield between the original yield map and each resampling technique. Figure 4-5 shows the comparison between the original yield map and the bilinear resampling method.

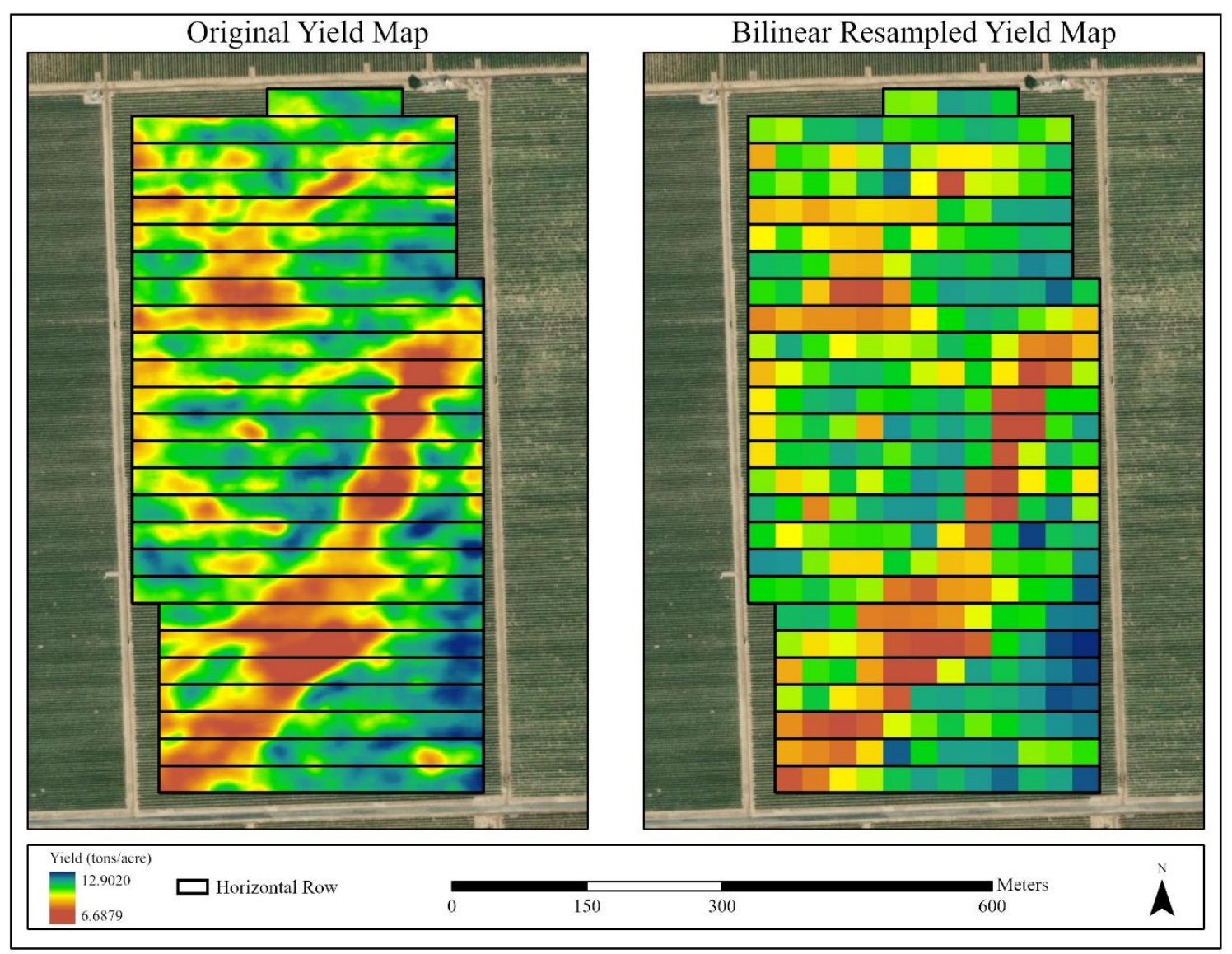

Figure 4-5: Vineyard Block Horizontal Row Comparison 
The mean yield of each row was calculated for the original yield map and the three resampled yield maps. The difference between the mean yield of the original rows and the three corresponding techniques was analyzed using the root mean square error (RMSE). The results determined that the bilinear technique produced the smallest error by a slim margin (Table 4). Although, the RMSE between all three techniques was small, it was important to choose the technique that minimized the impact of the scale effect. Therefore, the bilinear resampling technique was used to resample every available yield map to match the resolution of the Landsat imagery so they could be directly compared.

Table 4. Resampling Technique RMSE Result

\begin{tabular}{|l|l|}
\hline Resampling Technique & RMSE (tons/acre) \\
\hline Bilinear & 0.0988 \\
Nearest & 0.0993 \\
Cubic & 0.1004 \\
\hline
\end{tabular}

\subsubsection{Landsat 8 Imagery}

The final set of data that required adjusting was the Landsat 8 imagery. Before any image analysis could begin, each Landsat scene was clipped to the boundaries of the study area to increase processing efficiency. For this project, the normalized difference vegetation index (NDVI) and triangular vegetation index (TVI) were chosen based on research presented in Chapter 2. A script was then developed to iterate through the available Landsat 8 imagery to create separate NDVI and TVI images. The NDVI imagery was calculated using Equation 2-2 displayed in Section 2.2, while the TVI calculation (Equation 2-3) was adjusted to

$$
\mathrm{TVI}=0.5\left(95\left(R_{\mathrm{NIR}}-R_{\mathrm{Green}}\right)-305\left(R_{\mathrm{Red}}-R_{\mathrm{Green}}\right)\right)
$$

in order to account for the differences in the spectral bands of Landsat 8 versus the spectral bands that were originally used in Broge and Leblanc (2000). Overall, these two vegetation indices were analyzed to determine if one index located more accurate samples than the other.

\subsection{Summary}

A well designed database is a key component of a successful project. The design process started with mapping the relationships of the entities displayed in the conceptual model. Next, the logical data model was built to determine what attributes were needed in the final sample solution files. Once the database structure was designed, the datasets were cleaned and edited before implementation began. In the next chapter, the GIS and remote sensing techniques used in this project are documented 



\section{Chapter 5 - Implementation}

This chapter marks the beginning of the GIS and remote sensing analyses that resulted from the planning documented in the previous chapters. The analyses implemented within this project can be broken down into four sections: yield and imagery correlation analysis, Sample Locator Tool implementation, sample location yield extraction, and yield estimation error calculation. These four components were used to analyze the yield maps and imagery to output sample locations with their corresponding yield estimates. These yield estimations derived from each data source were used to address the clients' goal of determining if the methods used in the Sample Locator Tool were acceptable.

\subsection{Yield Map and Imagery Correlation Analysis}

The initial database of the normalized difference vegetation index (NDVI) and the triangular vegetation index (TVI) imagery was relatively large as it consisted of images from March to October for 2016, 2017, and 2018. Similar to research described in Sun et al. (2017), a correlation analysis was completed to better understand the temporal relationship between vineyard yield and vegetation indices. Within this project, pixel-bypixel correlation analyses were done between the resampled yield maps, NDVI, and TVI imagery. The objective of these analyses was to reduce the number of images processed by the Sample Locator Tool. The months of the imagery that resulted in the strongest correlations with the yield maps would be used for the Sample Locator Tool. The correlation process was broken down into three steps: create a Landsat pixel-by-pixel boundary, extract the raster values using the boundary file, and run a correlation analyses with the extracted raster values.

\subsubsection{Landsat Pixel Block Polygon Creation}

For these correlation analyses, four individual blocks were chosen from the study area. Landsat pixel-by-pixel polygons were created to provide a way to extract individual pixel values from the images. They were created by first clipping a Landsat 8 image to each block boundary. With the image clipped to the bounds of the block of interest, it was then converted to a polygon using the Raster to Polygon geoprocessing tool. The result of the tool was a polygon with separate boxes used to extract a raster value for each pixel. An example of the creation of a single Landsat pixel-by-pixel boundary can be seen in Figure 5-1. 


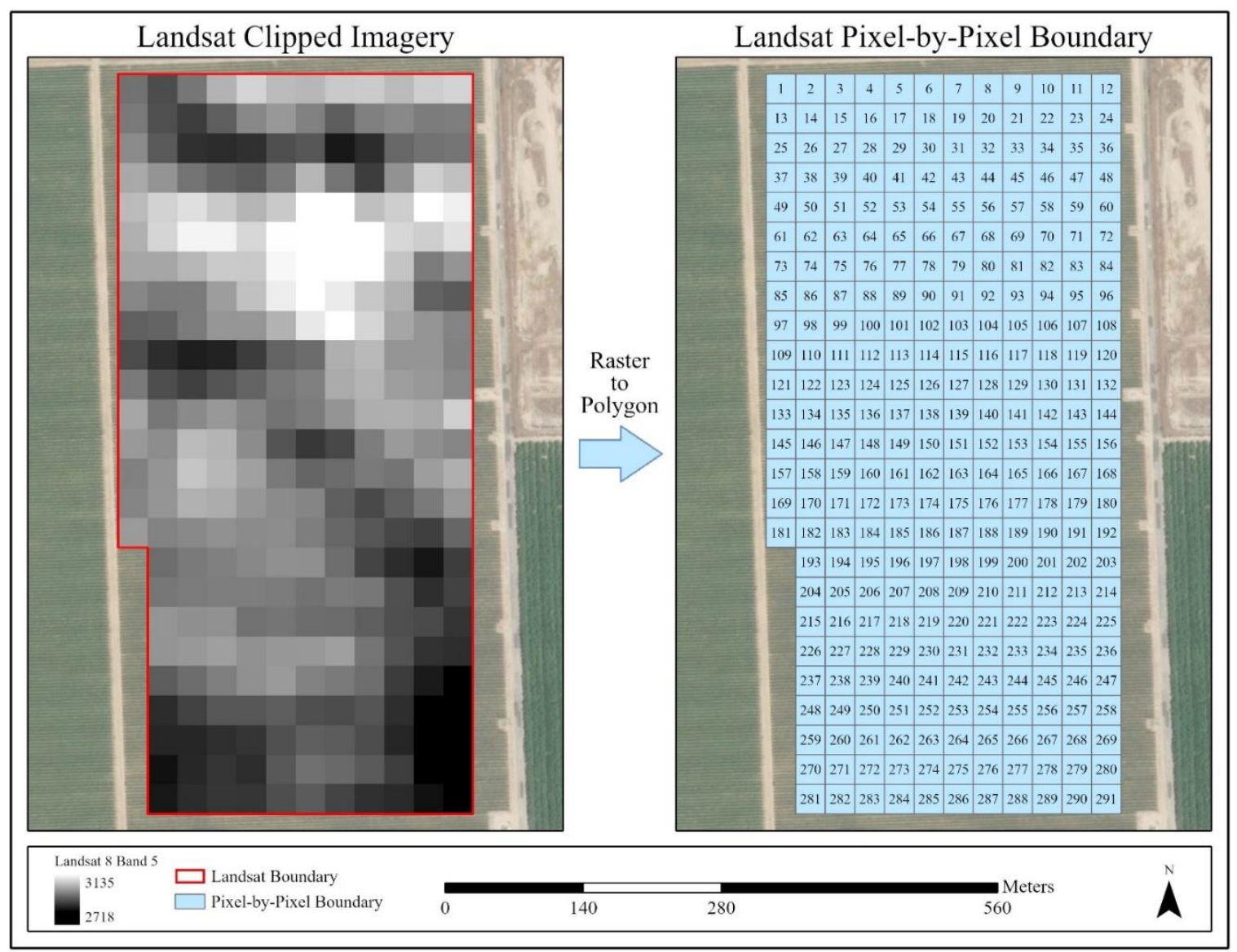

Figure 5-1: Landsat Pixel Block Boundary Creation

After the four Landsat pixel-by-pixel boundaries were created, each pixel within the boundaries was given its own identifier. This identifier attribute was used to ensure that the values being extracted from the rasters corresponded to the correct location of the Landsat pixel-by-pixel file.

\subsubsection{Extract Yield, NDVI, and TVI Values}

With the pixel block boundaries completed, the next step was to utilize these polygons to extract the yield, NDVI, and TVI raster values. A Python script was written to automate the process of extracting these raster values. The script first iterated through a chosen folder of NDVI or TVI imagery. For each folder, the script then used the Landsat pixelby-pixel boundary to extract the imagery values using the Zonal Statistics as Table geoprocessing tool. The table file that was output from the zonal statistics tool was then converted to an Excel spreadsheet using the Table to Excel geoprocessing tool. The result of this process were Excel files for every date an image was available that recorded the pixel identifier and its corresponding NDVI and TVI value. A visualization of this process for one NDVI image can be seen in Figure 5-2. 


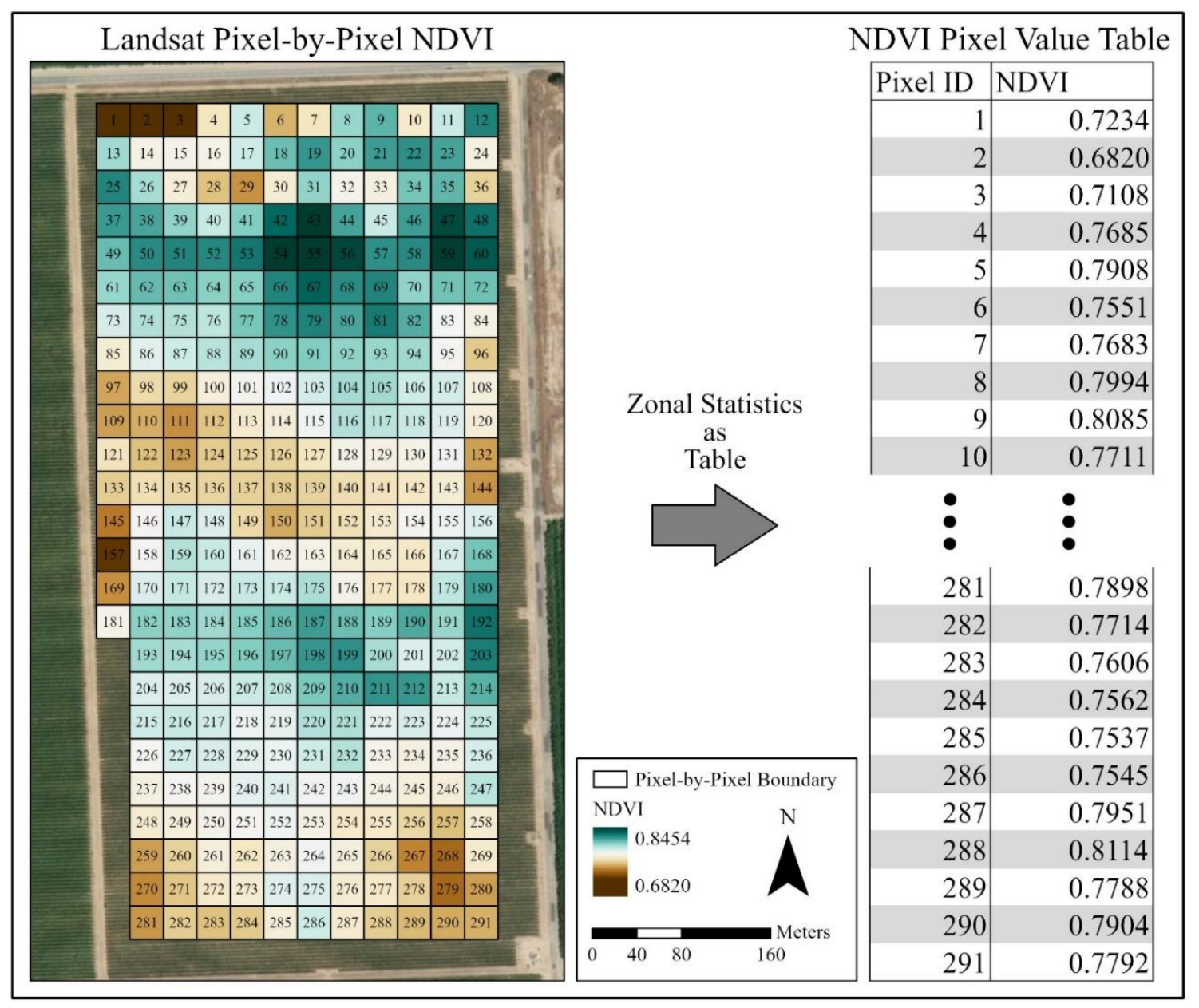

Figure 5-2: Landsat Pixel Value Extraction

The vineyard block shown in Figure 5-2 recorded 291 individual pixel values for each yield map, NDVI, and TVI raster available. The same process was done for the three remaining vineyard blocks. After all of the yield maps and remote imagery were processed for the four blocks, the pixel-by-pixel raster information from the spreadsheets were compiled into one large Excel file.

\subsubsection{Pixel-By-Pixel Correlation Analysis}

Once the yield, NDVI, and TVI pixel values were compiled into one Excel file, the final step was to perform correlation analyses. The focus of these analyses was to determine the strength of the correlation between a set of yield maps and the previous year's NDVI and TVI imagery for each block. An example would be calculating the correlation between the 2017 yield maps with their corresponding 2016 NDVI and TVI imagery. Using imagery from the previous year allows sample solutions to be located before the current harvest season begins, which allows additional time to establish sampling procedures. Therefore, the relationship between the yield maps and the previous year's imagery was analyzed instead of imagery from the same year. 
The Pearson correlation coefficient ( $r$ ) was calculated between each block's yield maps and the remote imagery from the previous year to measure the strength of their linear relationship. Table 5 displays the 2018 and 2017 yield map correlations with their previous year NDVI and TVI imagery for one of the pixel-by-pixel boundary files. The highlighted correlation coefficient in each column identifies the peak correlation coefficient for each vegetation index (NDVI and TVI). For this specific block, the imagery from July and August of the previous year had the strongest correlation coefficient with the yield maps.

Table 5. Pixel-by-Pixel Correlation Coefficient Results Example

\begin{tabular}{|r|c|c|}
\hline \multicolumn{3}{|c|}{ 2017 Yield Map Correlation $(r)$} \\
\hline Image Date & NDVI & TVI \\
\hline $3 / 16 / 2016$ & 0.1703 & 0.1925 \\
$4 / 1 / 2016$ & 0.1886 & 0.1541 \\
$4 / 17 / 2016$ & 0.0032 & 0.0124 \\
$5 / 19 / 2016$ & 0.1018 & 0.1481 \\
$6 / 20 / 2016$ & 0.1074 & 0.0794 \\
$7 / 6 / 2016$ & 0.5817 & 0.6558 \\
$7 / 22 / 2016$ & 0.6738 & 0.6695 \\
$8 / 7 / 2016$ & 0.6706 & 0.6743 \\
$8 / 23 / 2016$ & 0.6712 & 0.6398 \\
$9 / 8 / 2016$ & 0.6471 & 0.6636 \\
$10 / 10 / 2016$ & 0.3727 & 0.1390 \\
$10 / 26 / 2016$ & 0.3727 & 0.1390 \\
\hline
\end{tabular}

\begin{tabular}{|r|l|l|}
\hline \multicolumn{2}{|c|}{ 2018 Yield Map Correlation $(r)$} \\
\hline Image Date & NDVI & TVI \\
\hline $3 / 3 / 2017$ & 0.0049 & 0.0025 \\
$4 / 4 / 2017$ & 0.0755 & 0.0263 \\
$4 / 20 / 2017$ & 0.2148 & 0.1769 \\
$5 / 6 / 2017$ & 0.2899 & 0.4049 \\
$5 / 22 / 2017$ & 0.2466 & 0.2461 \\
$6 / 7 / 2017$ & 0.3560 & 0.3875 \\
$6 / 23 / 2017$ & 0.4303 & 0.4352 \\
$7 / 9 / 2017$ & 0.5076 & 0.4765 \\
$7 / 25 / 2017$ & 0.5316 & 0.4843 \\
$8 / 10 / 2017$ & 0.5089 & 0.4689 \\
$8 / 26 / 2017$ & 0.5136 & 0.5041 \\
$9 / 11 / 2017$ & 0.4516 & 0.3865 \\
$9 / 27 / 2017$ & 0.4204 & 0.4212 \\
$10 / 13 / 2017$ & 0.2515 & 0.2665 \\
$10 / 29 / 2017$ & 0.0981 & 0.2290 \\
\hline
\end{tabular}

The combined results of the four chosen blocks showed that the peak correlation coefficient occurred in July and August for $75 \%$ of the measured blocks for every year analyzed. Therefore, only imagery from July and August were used in the following analyses. Applying imagery from previous years to estimate yield has been a technique presented in previous research including Cunha et al. (2010) and Meyers et al. (2020).

One inconclusive outcome from these analyses was the comparison between NDVI and TVI imagery. The results showed that neither vegetation index consistently showed stronger correlation coefficients than the other. Therefore, both NDVI and TVI imagery from July and August were used as the main sources of data for the Sample Locator Tool.

\subsection{Sample Locator Tool}

The primary focus of this project was the creation of the Sample Locator Tool. E. \& J. Gallo Winery's Viticulture Research Department have had success estimating grape 
maturity using a single vine row solution derived from the methods discussed in Meyers et al. (2020). The clients wanted to determine if this method was a viable option for locating samples to estimate yield. The first objective of these tools was to determine if satellite imagery could be used to help locate sampling solutions to estimate yield. The secondary objective was to determine if NDVI or TVI imagery produced more accurate sample locations. The process of creating the Sample Locator Tool was divided into three steps: design, scripting, and output.

\subsubsection{Sample Locator Tool Design}

Before the Python script was written, a flowchart of the tool's main functions was created. The purpose of this flowchart was to visualize how the tool would process the original data to create the sample solution locations. The flowchart diagram used to plan the Sample Locator Tool's main functions is displayed below in Figure 5-3.

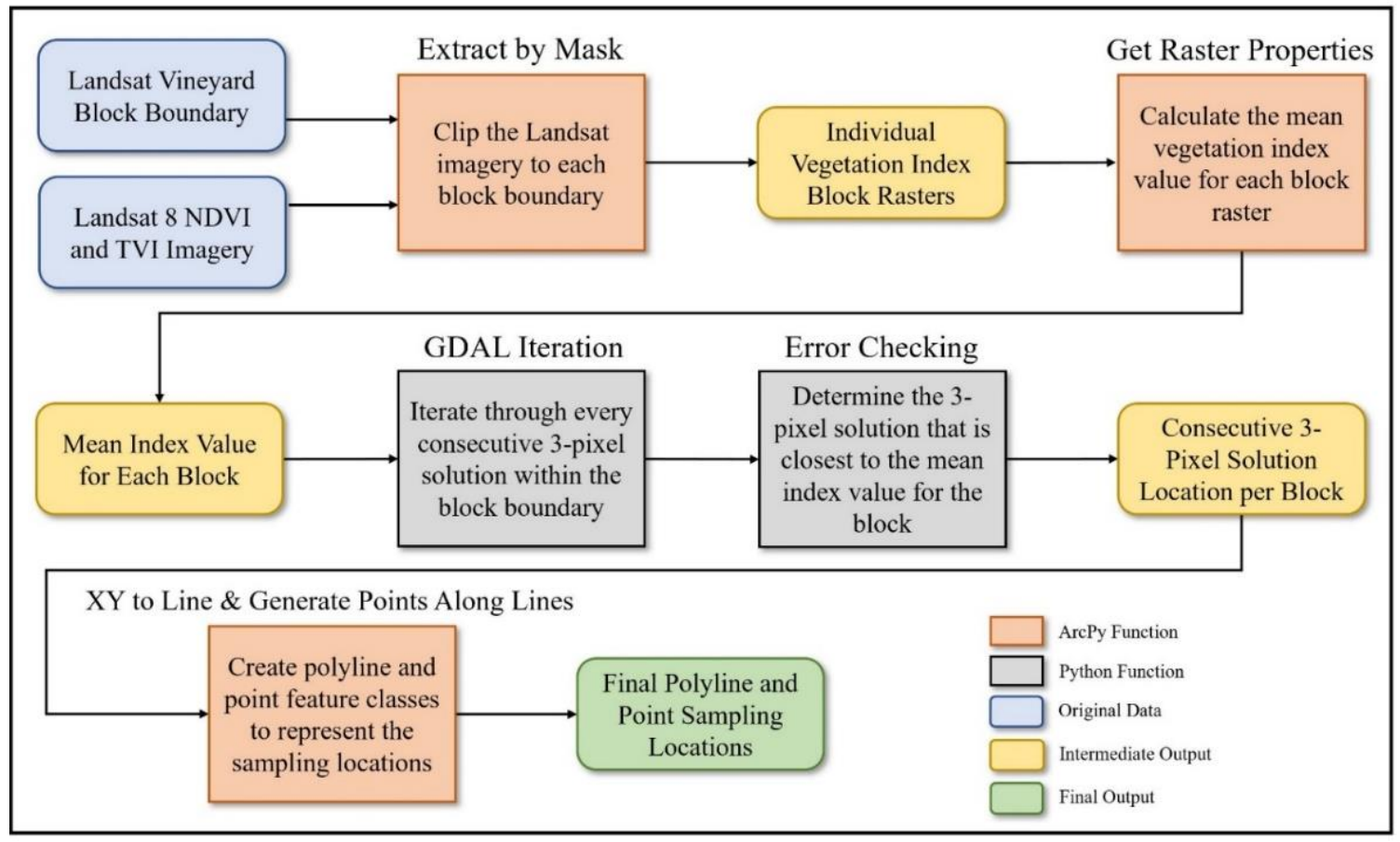

Figure 5-3: Sample Locator Tool Flowchart

The process of this tool began by taking one NDVI image and clipping it to the Landsat 8 boundary file using the Extract by Mask geoprocessing tool, resulting in an individual raster for each vineyard block. Next, the tool calculated the mean NDVI value for each raster using the Get Raster Properties geoprocessing tool. After each block's mean NDVI was known, each respective raster was analyzed three consecutive pixels at a time to determine the location that best matched the mean. Figure 5-4 demonstrates the two different approaches used to analyze the three consecutive pixels. Depending on the vine angle of the block, the three pixels were analyzed vertically or horizontally. If the vines were facing north to south the pixels were analyzed vertically, while east to west vines were analyzed horizontally. Each possible 3-pixel solution was checked before the final location of the sample solution was chosen. 


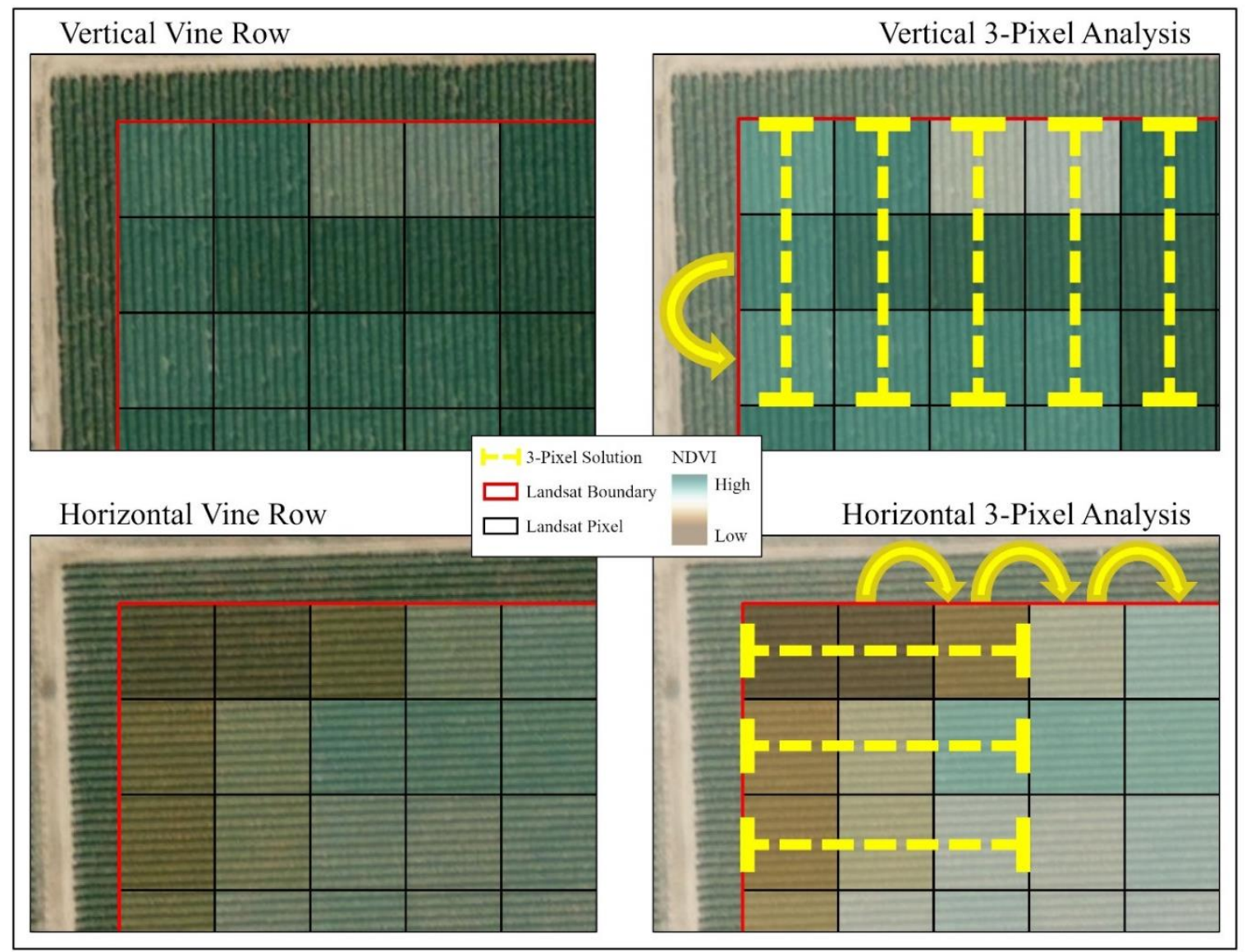

Figure 5-4: Comparison of Analysis Between Horizontal and Vertical Vine Angle

After every possible solution was checked based on the vine angle, the three pixels with an average NDVI closest to the average NDVI of the entire block was chosen as the final solution location. Once the 3-pixel solution was identified, the tool then created two separate sample location outputs: a 90 meter polyline and a 20 point solution. Once the functions of the tool were well defined, the next step was to automate the functions within a Python script.

\subsubsection{Python Scripting}

The objective of writing the Python script was to automate all of the geoprocessing tools depicted in Figure 5-3. The PyScripter integrated development environment (IDE) was chosen to write and test the Sample Locator Tool due to its ease of use. To accomplish the primary functions of the tools, the ArcPy, Geospatial Data Abstraction Library (GDAL), and NumPy Python libraries were used. The ArcPy library was used to gain access to a wide range of Esri geoprocessing tools. The GDAL and NumPy libraries were required to open, read, and analyze the raster files. Overall, many iterations of the tool were scripted until the outputs matched the intended outcome. The final Sample Locator Tool's Python script is shown in Appendix A. 


\subsubsection{Sample Locator Tool Output}

The last process of implementing the Sample Locator Tool was to check its sample solution outputs. While the tool was written, the sample solutions were checked to determine that they were in the correct location and formatted correctly. To determine that the solutions were in the correct location, a manual check was done to ensure the three pixels that overlapped with the sample solution were the correct values. The format of the sample solutions was also checked to verify that they overlapped the pixels with the correct vine angle. As described in the design section, there were sample solution files created for each vineyard block and image. Figure 5-5 shows an example of the types of sample solutions output by the tool for an individual block.

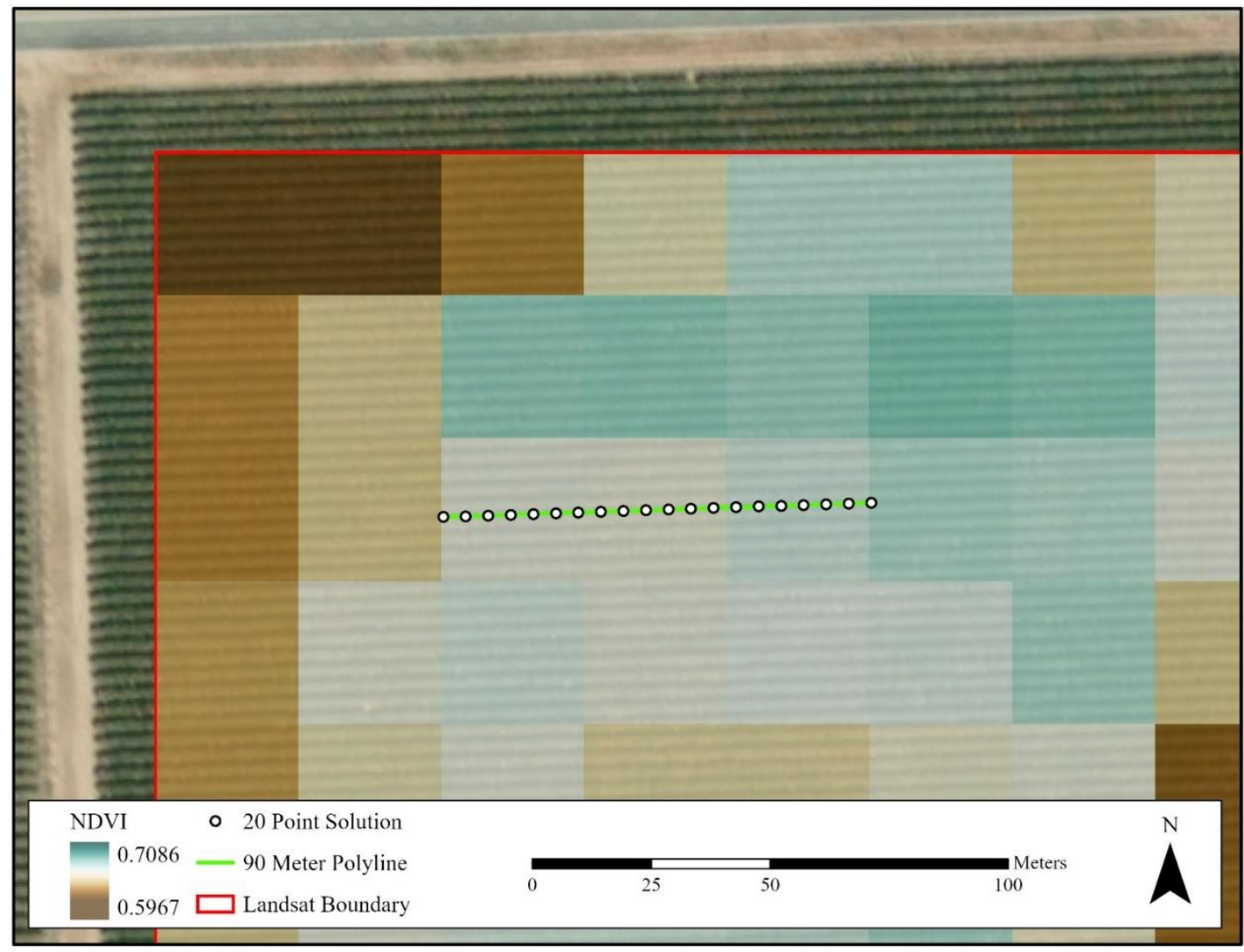

Figure 5-5: 90 Meter Polyline and 20 Point Solution Example

The 90 meter polyline intersects all three pixels while matching the vine angle of the block. The sample location starts at the center of the edge of the first pixel, or 15 meters from the corresponding corners. This solution represents the visual guide the Viticulture Research Department used to show the samplers where to collect clusters of grapes in the field. For this project, an additional point feature class was created that contained 20 points evenly spaced across the 90 meter polyline. These points were used to extract values from the yield maps similar to the way a field sampler would extract 20 grape clusters in the vineyard. After a subset of the sample solutions were manually checked for their positional accuracy and format, two final datasets were needed to check the validity of these solutions as explained in the next section. 


\subsubsection{Random Sample Locations}

To test the effectiveness of the sample solutions output by the tools, two random sample solution files were created as the control group. The first file consisted of 20 randomly generated points within each vineyard block boundary. These points were created using the Create Random Points geoprocessing tool and the vineyard block boundary as a way to constrain the 20 points to each block. This random 20 point file was included in these analyses because the Viticulture Research Department have implemented this sampling method as a control group in previous research. The second file is a randomly generated single vine row solution. The format is the same as the 20 point solution described in Section 5.2.3, where 20 sampling points are evenly spaced out on a 90 meter polyline. However, the location of the polyline was randomized to create another control group to test the Sample Locator Tool's outputs. After all the required sample solutions were created, the next step was to extract the corresponding values from the yield maps to create a yield estimate for each sample location.

\subsection{Yield Extraction Tool}

The third step of the GIS and remote sensing implementation was to extract the yield values using the sample solutions output from the locator tool and the randomly generated samples. The values extracted from the yield maps using the sample solutions were used to summarize their corresponding yield estimates. The Python script for this tool used the same libraries as the Sample Locator Tool described in Section 5.2.2. Therefore, the Yield Extraction Tool overview only focuses on the tool's design and output.

\subsubsection{Yield Extraction Tool Design}

Another flowchart was created to help organize the necessary functions of the Yield Extraction Tool. More specifically, the ArcPy and Python functions were listed to determine how the tool would analyze the sample solutions and the yield maps to output yield estimation tables. Figure 5-6 displays the relationships and processes designed for the Yield Extraction Tool.

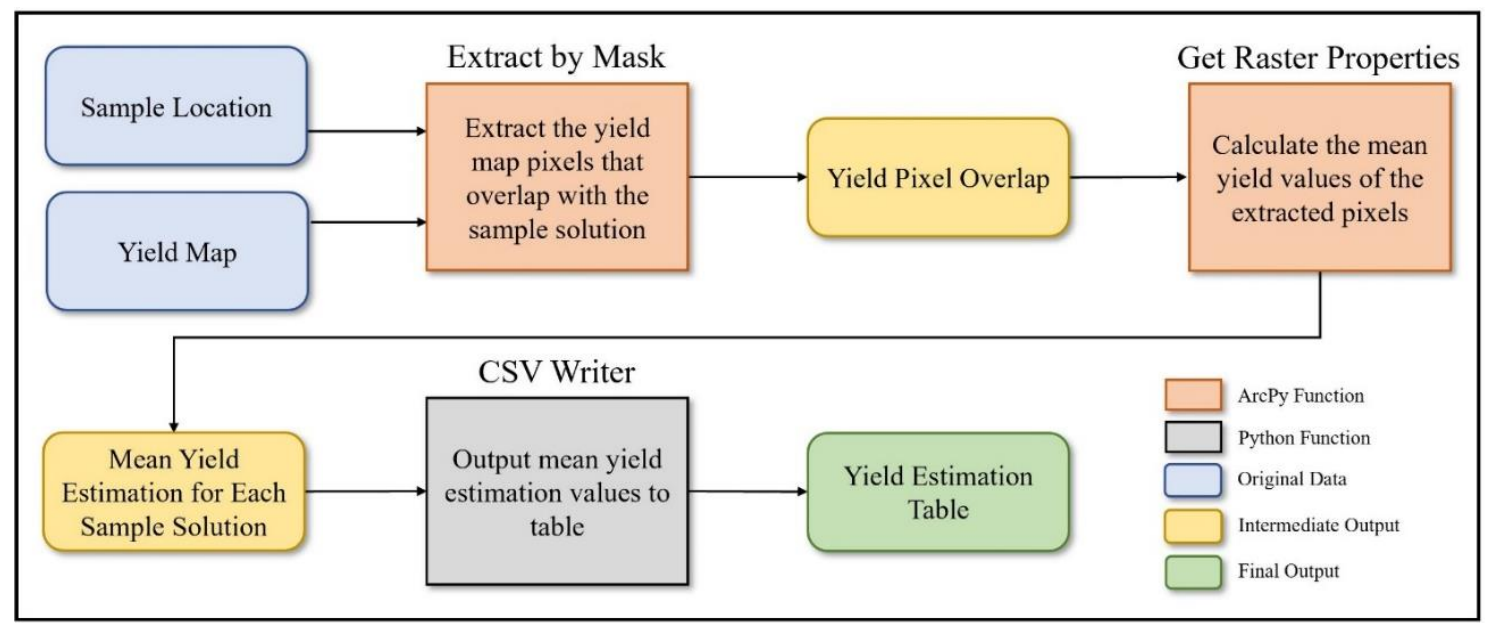

Figure 5-6: Yield Extraction Tool Flowchart 
The Yield Extraction Tool first iterated through the sample locations with their corresponding yield maps. The Extract by Mask geoprocessing tool was used to extract the yield map pixels that overlapped with the sample solution. Once the overlapping pixels were masked, the mean yield of these pixels were calculated with the Get Raster Properties geoprocessing tool. The mean yield value was treated as the yield estimation for the given sample solution. This value was then stored alongside all of the yield estimations for every block within the study area for the given raster.

Through extensive collaboration with the clients, many iterations of the yield extraction design were explored. Initially, the plan was to use the 90 meter polyline solution to extract yield values from the resampled yield maps. However, it was determined that better results would occur if the original yield maps were used. Thus, avoiding any possible artifacts or aggregation issues present in the resampled yield maps. The second edit made to the extraction methods involved the use of the 90 meter polyline solution. When samples are taken in the field, 20 clusters of grapes are taken evenly spaced along the 90 meter line. To simulate this sampling technique, the 20 point solution was used to extract yield values instead of the 90 meter polyline.

Since the time frame of this project did not allow for samples to be collected in the field, it was important to use an extraction method that most accurately resembled what occurs in the field. Overall, the extraction methods used in this tool were developed to match the field sampling protocol used by the Viticulture Research Department.

\subsubsection{Yield Extraction Tool Output}

While the Python script was being written for the Yield Extraction Tool, the outputs were verified to ensure the correct values were derived. For every sample solution a yield estimation was extracted from the yield map. Figure 5-7 shows an example of a single block with its extracted yield map pixels and final yield estimation. 


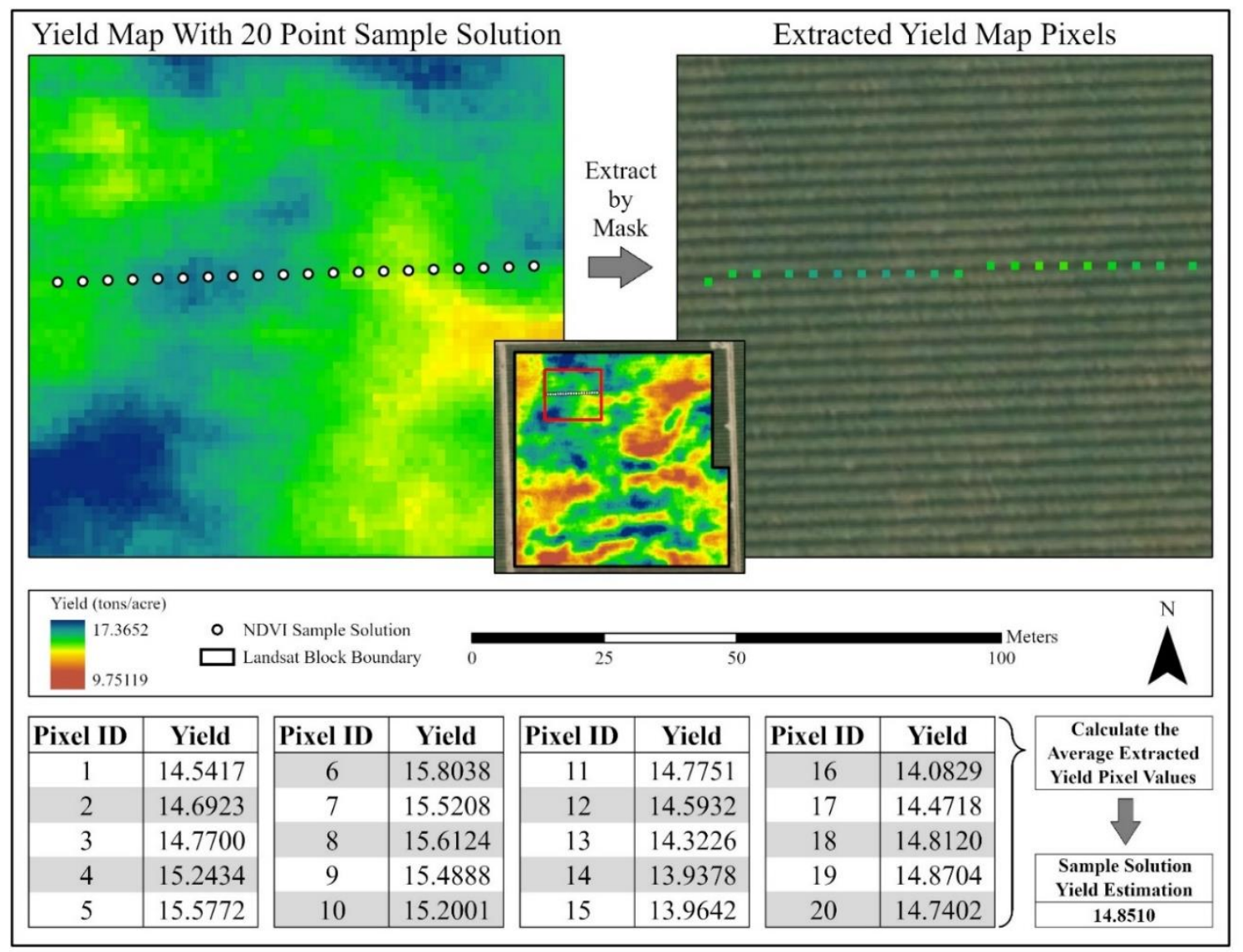

Figure 5-7: Sample Solution Extracted Yield Map Pixels and Yield Estimate

Figure 5-7 visualizes the procedures of the Yield Extraction Tool displayed in Figure 5-6. First, the vineyard block's 20 point sample solution was used to extract the overlapping yield map pixels. The Extract by Mask geoprocessing tool then calculated the average of the extracted pixels. This average value of 14.8510 tons per acre was used as this solution's yield estimation.

A subset of the yield estimates derived from the tool were manually checked to ensure the correct pixel values were being summarized. After the yield estimates from the subset were verified, all of the sample solutions were run through the Yield Extraction Tool, which is displayed in Appendix B.

\subsection{Yield Estimation Error Analysis}

The final step of the GIS and remote sensing implementation was to calculate the yield estimation errors. For every raster input, 36 separate sample locations were produced. Before the sample locations could be directly compared, a yield estimation error was calculated for each sample. These error values were used to determine the accuracy of each sample. A breakdown of these error analyses was completed and is described in the sections below.

For this project, the sample location error was defined as the difference between the sample's yield estimation and the mean yield of the original yield map. Both values were 
previously calculated in the implementation process. The yield estimation values for every sample was output into a spreadsheet by the Yield Extraction Tool. The mean yield of every original yield map was logged in a separate spreadsheet by the Sample Locator Tool. Together, these values would help define the accuracy of every sample location. Figure 5-8 shows a single vineyard block's 2017 yield map and its corresponding 2016 sample locations.

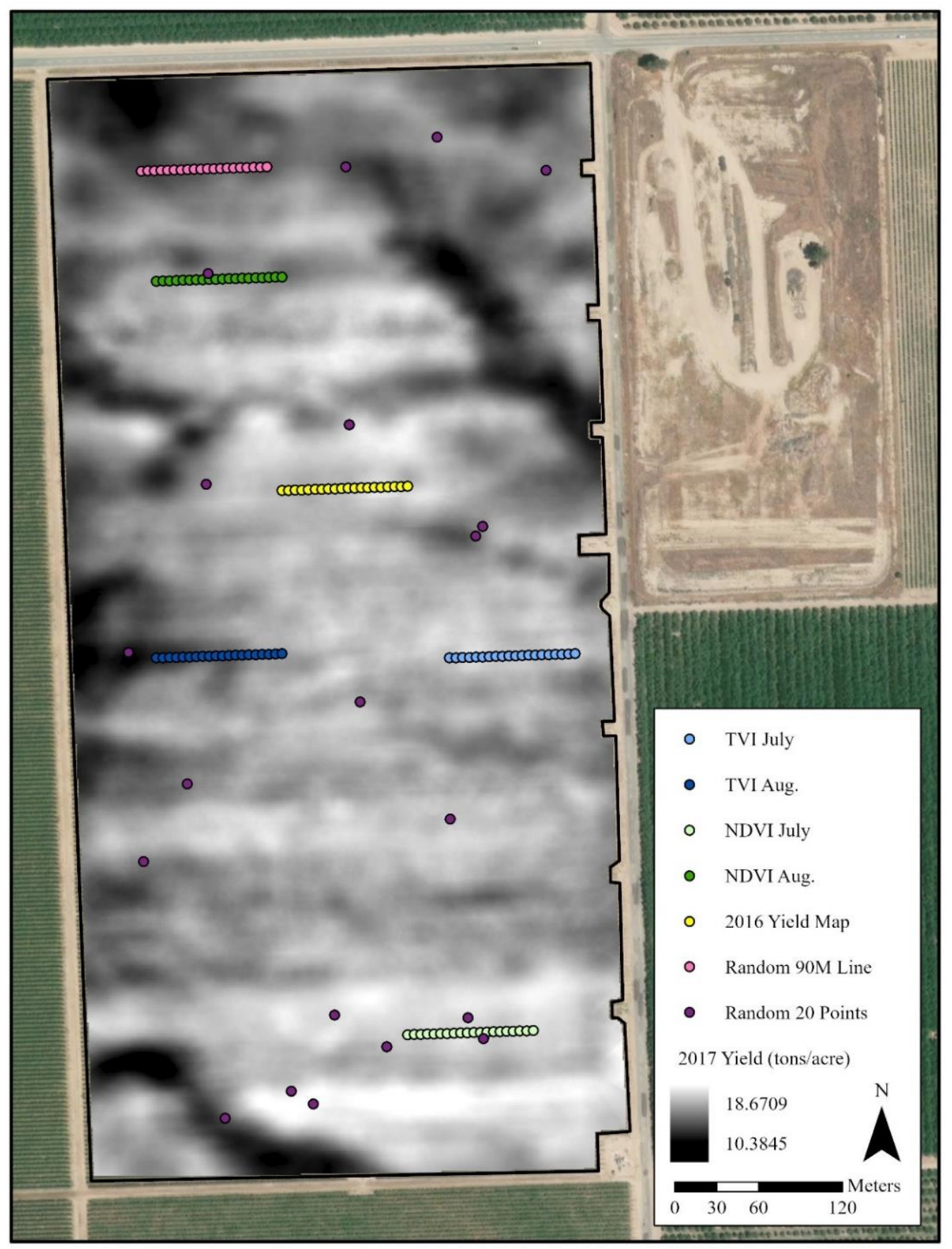

Figure 5-8: Example of 2016 Sample Locations with 2017 Yield Map 
Figure 5-8 shows a single vineyard block and its corresponding sample solutions. All of the sample locations are along a single 90 meter vine row with the exception of the Random 20 Points, which is only confined to the boundary of the block. The vineyard block's corresponding yield estimations and estimation errors for each solution is displayed in Table 6.

Table 6. Extracted Yield Estimations and Yield Errors for Figure 5-8

\section{Original Mean Yield (tons/acre)}

15.2565

\begin{tabular}{|l|rrrrrrr|}
\hline & $\begin{array}{r}2016 \\
\text { Yield Map }\end{array}$ & $\begin{array}{r}\text { July } \\
\text { NDVI }\end{array}$ & $\begin{array}{r}\text { Aug. } \\
\text { NDVI }\end{array}$ & $\begin{array}{r}\text { July } \\
\text { TVI }\end{array}$ & $\begin{array}{r}\text { Aug. } \\
\text { TVI }\end{array}$ & $\begin{array}{r}\text { Random } \\
\text { 20 Points }\end{array}$ & $\begin{array}{r}\text { Random } \\
\text { 90M Vine }\end{array}$ \\
\hline $\begin{array}{l}\text { Yield Estimate } \\
\text { (tons/acre) }\end{array}$ & 16.3508 & 15.7808 & 15.1768 & 16.7655 & 15.3055 & 15.4224 & 13.5771 \\
$\begin{array}{l}\text { Yield Error } \\
\text { (tons/acre) }\end{array}$ & 1.0943 & 0.5243 & 0.0797 & 1.5090 & 0.0490 & 0.1659 & 1.6794 \\
\hline
\end{tabular}

Table 6 displays how each yield error was calculated by finding the difference between the mean of the original yield map and each yield estimate. The results for this specific vineyard block show that the sample location derived from the August TVI imagery produced the smallest yield error of 0.0490 tons per acre. Therefore, the sample solution derived from the August TVI imagery produced the most accurate yield estimation in this example. The yield error calculations for the remaining 35 vineyard blocks in the study area were also calculated, and the results are summarized in the following chapter.

\subsection{Summary}

The GIS and remote sensing implementation for this project resulted in four separate processes. The first process resulted in a tool that conducted pixel-by-pixel correlation analyses between yield and vegetation indices to determine the date of the imagery that should be used. Next, the Sample Locator Tool identified optimal sampling locations using NDVI and TVI imagery. The third process involved implementing the Yield Extractor Tool which calculated the yield estimates for each sample location. Finally, the yield estimation errors were calculated to summarize the accuracy of each sample location. A summary of the yield estimation error results is documented in the following chapter. 


\section{Chapter 6 - Results and Analysis}

This chapter summarizes the results and analysis derived from the planning and implementation discussed in the previous chapters. The goal of this project was to determine if satellite imagery could identify efficient sampling locations used to estimate vineyard yield accurately. This inquiry was addressed through comparing the yield estimation errors of each sample location derived from the Sample Locator Tool.

Within Section 6.1, the results of the yield estimation errors were reviewed. Section 6.2 highlights a discussion of some limitations in this project. The final section of this chapter wraps up the results and analyses organized to address the Viticulture Research Department's goal.

\subsection{Yield Estimation Error Results}

The yield estimation error results were determined through the tools presented in Chapter 5. This overview begins by listing the data categories of the sample location files. Next, estimation results are given by displaying and interpreting the sample estimation errors for 2017 and 2018 across every category. Finally, this section concludes with a comparison between NDVI and TVI yield estimation errors.

\subsubsection{Tested Data Categories}

There were seven separate sample location categories analyzed within this project. Each of the sample location categories were derived from yield maps, Landsat 8 imagery, or random generator geoprocessing tools. Table 7 lists these categories and shows if they are created from the Sample Locator Tool or randomly generated.

\section{Table 7. Sample Location Categories}

\begin{tabular}{|l|l|}
\hline Sample Location Category & Creation Method \\
\hline Resampled Yield Map & Sample Locator Tool \\
July NDVI & Sample Locator Tool \\
August TVI & Sample Locator Tool \\
July TVI & Sample Locator Tool \\
August TVI & Sample Locator Tool \\
Random 20 Points & Randomly Generated \\
Random 90m Vine Row & Randomly Generated \\
\hline
\end{tabular}

The first set of samples were derived from the previous year's resampled yield maps. Using yield maps as an input for the Sample Locator Tool was an interesting approach to test how well yield from previous harvests could estimate the current harvest's yield. However, it should be noted that yield maps are not a readily available resource for most vineyard managers. Therefore, satellite imagery was the main point of focus for this project due to its availability for any vineyard of interest. 
The next set of rasters analyzed was the NDVI imagery from July and August for 2017 and 2018. This vegetation index was chosen based on its popularity amongst previous viticulture research (Cunha et al., 2010; Meyers et al., 2020; Sun et al., 2017 ). To test another vegetation index, the TVI was chosen based on its success estimating yield in research documented by Hall and Wilson (2013). Together, the NDVI and TVI made up the two vegetation indices tested within this project.

Without a benchmark or control group, the results from the imagery would be difficult to assess. Therefore, two randomly generated samples were created as reference points to compare to the solutions derived from the yield maps and vegetation indices. Together, the sample locations derived from these seven categories were compared to determine if the samples created from the imagery presented a viable option to estimate yield.

\subsubsection{Yield Estimation Error Results}

The first set of sample estimation errors that were evaluated were for the 2017 harvest season. In this instance, data from 2016 were processed by the Sample Locator Tool to output samples to estimate the 2017 yield. Within the study area, there were 36 individual vineyard blocks. Therefore, every sample location category contained 36 yield estimation error values. To compare the accuracy of the sample location categories, the RMSE of their corresponding 36 yield estimation errors was calculated. Figure 6-1 shows an example of a sample location category and its 36 corresponding sample locations used to calculate its RMSE.

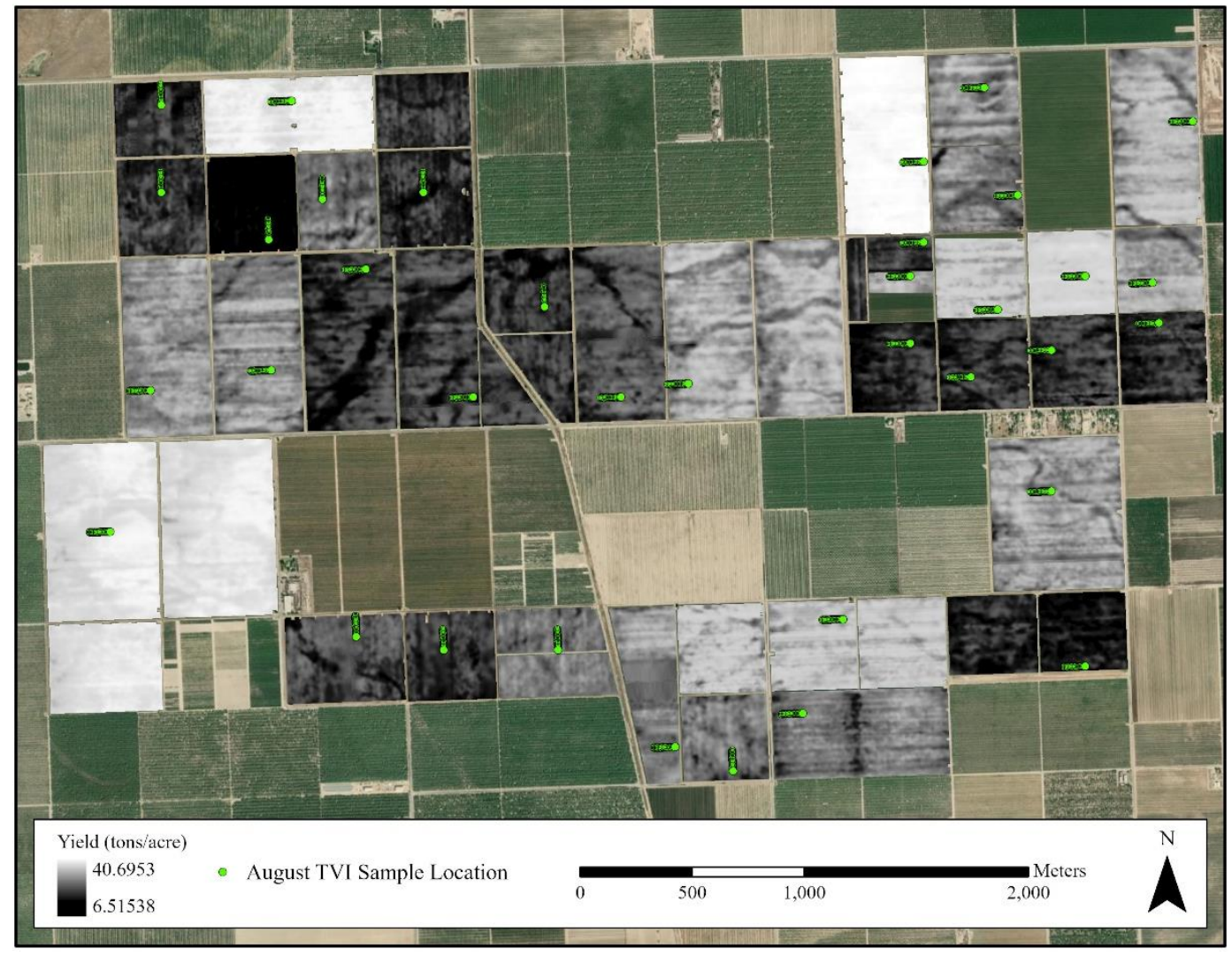

Figure 6-1: August 2016 TVI Solutions with Corresponding 2017 Yield Maps 
As displayed in Figure 6-1, the RMSE was calculated using the 36 sample locations generated by the TVI imagery from August 2016. In this example, the RMSE of the 36 samples generated from the August TVI image was 0.9021 tons per acre. The yield estimation error result for the August TVI image and the remaining six categories are shown and ranked in Table 8.

\section{Table 8. 2017 Yield Estimation Category RMSE Ranking}

\begin{tabular}{|c|l|c|}
\hline \multicolumn{2}{|c|}{ 2017 Yield Estimation RMSE } \\
\hline Rank & \multicolumn{1}{|c|}{ Category } & RMSE (tons/acre) \\
\hline 1 & Random 20 Points & 0.2305 \\
2 & Random 90m Vine Row & 0.7954 \\
3 & Aug. NDVI & 0.8685 \\
4 & 2016 Yield Map & 0.8748 \\
5 & Aug. TVI & 0.9021 \\
6 & July TVI & 0.9789 \\
7 & July NDVI & 1.0248 \\
\hline
\end{tabular}

According to Table 8, both of the randomly generated sample solutions resulted in the lowest yield estimation RMSE for the 2017 harvest season. The Random 20 Points was clearly the best performing set of sample solutions of the seven sample location categories. The remaining six categories (see Table 7), had similar performing RMSE values. Therefore, paired t-tests were calculated between each category to determine if there were statistically significant differences between each group's 36 yield estimation errors. The results of the paired t-tests are presented in Table 9.

Table 9. 2017 Yield Estimation Paired t-test Results

\begin{tabular}{|l|c|c|c|c|c|c|}
\hline \multicolumn{7}{|c|}{2017 Yield Estimated by 2016 Data: Paired t-test (p-value) } \\
\hline & 2016 Yield Map & \multicolumn{1}{c|}{ Random 20 Pts. } & \multicolumn{1}{c|}{ Random 90m Vine } & \multicolumn{1}{c|}{ July NDVI } & \multicolumn{1}{c|}{ Aug. NDVI } & July TVI \\
\hline \multirow{2}{*}{$\begin{array}{l}\text { Random 20 Points } \\
\text { Random 90m Vine }\end{array}$} & 0.0003 & - & - & - & - & - \\
\cline { 2 - 7 } $\begin{array}{l}\text { July NDVI } \\
\text { Aug. NDVI }\end{array}$ & 0.8596 & $4.59 \mathrm{E}-05$ & - & - & - & - \\
\cline { 2 - 8 } $\begin{array}{l}\text { July TVI } \\
\text { Aug. TVI }\end{array}$ & 0.3850 & $9.33 \mathrm{E}-10$ & 0.2571 & - & - & - \\
\cline { 2 - 8 } & 0.9966 & $7.08 \mathrm{E}-08$ & 0.8346 & 0.2144 & - & - \\
\cline { 2 - 8 } & 0.4117 & $6.76 \mathrm{E}-06$ & 0.4463 & 0.9892 & 0.2197 & - \\
\hline
\end{tabular}

A significance level of $\alpha=0.05$ was chosen for the paired t-tests. Accordingly, any p-value displayed in Table 9 less than 0.05 was considered to be statistically significant and was highlighted in green. In this application, a significant $\mathrm{p}$-value represented a substantial difference in yield estimation errors between the two categories measured.

In Table 9, the p-value between the yield estimation errors of the 2016 yield map and the Random 20 Points is represented by the cell with a value of 0.0003 . Thus, there was a statistically significant difference between the yield estimation errors of these two categories. By combining this information with the yield estimation error ranking listed in Table 8, it can be concluded that the Random 20 Points performed significantly better than the 2016 yield map. For this harvest year, the only statistically significant 
differences were between the Random 20 Points and the remaining six categories. In other words, the yield estimation accuracy of the sample solutions output by the NDVI imagery, TVI imagery, and Random 90 Meter Vine Row samples were inseparable. To check these results, the same ranking and t-test analyses were done for the 2018 harvest season's yield estimation errors.

\subsubsection{Yield Estimation Error Results}

The final year that was analyzed was the 2018 harvest season. The methods used to analyze these yield estimation errors were identical to the methods described for the 2017 analyses. The first component of the 2018 harvest analyses was the yield estimation error ranking, which is displayed in Table 10.

Table 10. 2018 Yield Estimation Category RMSE Ranking

\begin{tabular}{|c|l|c|}
\hline \multicolumn{2}{|c|}{2018 Yield Estimation RMSE } \\
\hline Rank & \multicolumn{1}{|c|}{ Category } & RMSE (tons/acre) \\
\hline 1 & Random 20 Points & 0.2630 \\
2 & 2017 Yield Map & 0.5687 \\
3 & Aug. NDVI & 0.6555 \\
4 & Aug. TVI & 0.7661 \\
5 & Random 90m Vine Row & 0.7722 \\
6 & July NDVI & 0.8076 \\
7 & July TVI & 0.8347 \\
\hline
\end{tabular}

Once again, the best performing category was the Random 20 Points, which performed similarly to its corresponding 2017 yield estimation results shown in Table 8 . One of the key differences between the 2017 and 2018 yield estimation error results was the accuracy of the previous year's yield maps. When using the sample solutions derived from the 2016 yield maps to estimate the 2017 yield resulted in a RMSE of 0.8748 tons per acre (Table 8). However, using the 2017 yield maps solutions to predict the 2018 yield output a RMSE of 0.5687 tons per acre, which is more accurate than the previous year's results (Table 8).

The RMSE derived from the NDVI and TVI imagery were relatively similar for both years. In most cases, the sample locations created from the remote imagery performed worse than the randomly generated samples and yield map samples. To determine if the differences between the yield estimation errors for 2018 were significant, another set of paired t-test analyses was completed. The p-values generated from the paired t-tests are documented in Table 11. 
Table 11. 2018 Yield Estimation Paired t-test Results

\begin{tabular}{|c|c|c|c|c|c|c|}
\hline \multicolumn{7}{|c|}{2018 Yield Estimated by 2017 Data: Paired t-test (p-value) } \\
\hline & 2017 Yield Map & Random 20 Pts. & Random 90m Vine & July NDVI & Aug. NDVI & July TVI \\
\hline \multirow{6}{*}{$\begin{array}{l}\text { Random } 20 \text { Points } \\
\text { Random 90m Vine } \\
\text { July NDVI } \\
\text { Aug. NDVI } \\
\text { July TVI } \\
\text { Aug. TVI } \\
\end{array}$} & 0.0019 & - & - & - & - & - \\
\hline & 0.0873 & $6.59 \mathrm{E}-05$ & - & - & - & - \\
\hline & 0.0485 & $3.72 \mathrm{E}-07$ & 0.8941 & - & - & - \\
\hline & 0.0518 & $1.23 \mathrm{E}-08$ & 0.7216 & 0.4705 & - & - \\
\hline & 0.0386 & 4.29E-06 & 0.7483 & 0.7940 & 0.4578 & - \\
\hline & 0.0487 & 2.99E-06 & 0.9483 & 0.7663 & 0.7249 & 0.6103 \\
\hline
\end{tabular}

The results for the Random 20 Points category for 2018 was similar to the previous year. From the outcome of both years, it is clear that the Random 20 Points produced the smallest yield estimation error. However, sampling 20 random points within a vineyard block is inefficient as it usually requires the field sampler to traverse a majority of the block.

As shown by Table 10, there was a major improvement in accuracy of using the previous year's yield map. Table 11 verifies that this improvement resulted in statistically more accurate yield estimates compared to the sample locations created from the July NDVI, July TVI, and August TVI imagery. Even though the samples generated from the yield maps provided a significantly improved result, it is important to reiterate that yield maps are not an available resource for most growers. Therefore, vegetation indices derived from satellite imagery would be the ideal source of data due to its temporal and spatial availability.

\subsubsection{NDVI Versus TVI Yield Estimation}

One of the objectives of this project was to determine if there was a significant difference between sample locations derived from NDVI or TVI imagery. According to the ranking and t-test analyses performed for the 2017 and 2018 harvest seasons, there were no significant differences between the accuracy of the sample locations derived from both vegetation indices. Also, these sample locations were generally less accurate than the randomly generated samples and the yield map samples.

These results are similar to those of the pixel-by-pixel correlation analyses described in Section 5.1, which found no significant differences between the correlation of the NDVI and TVI imagery with the resampled yield maps. The various analyses performed within this project suggest that there were no meaningful differences in performance between the sample locations derived from the NDVI and TVI imagery. Overall, the results from this project suggest that the methods conducted within the Sample Locator Tool require adjusting and testing before any major implementation can occur.

\subsection{Discussion}

Although this project was completed as intended, there were two possible limitations that should be noted. The first limitation was the lack of field sampling done to test the samples output by the Sample Locator Tool. The second possible limitation was the use 
of Landsat 8 imagery and its spatial resolution. Both limitations are discussed in greater detail in the sections below.

\subsubsection{Field Sampling}

A majority of viticulture research projects usually implement some form of field sampling. Typically, these measurements help verify or reject the hypothesis being tested. The given time frame for this project determined that field sampling would not be a viable option. Therefore, the original yield maps provided by the Viticulture Research Department were used as the true vineyard yield. Even though these yield maps were the best replacement for field sampling, it is possible that the results from samples taken in the vineyard could differ from the results presented by the yield maps. Ultimately, future research will require sampling in the field to gain a better understanding of how well these sample locations perform.

\subsubsection{Landsat 8 Spatial Resolution}

The second possible limitation present in this project was the use of Landsat 8 imagery. One of its main drawbacks compared to other satellite programs is its 30 meter spatial resolution. A newer satellite program that has been used alongside or in place of Landsat 8 is the Sentinel 2 satellite program. The benefit of using Sentinel 2 imagery for this project would be its higher spatial resolution of 10 meters. It is possible that with higher resolution imagery, more precise decisions can be made to identify where samples should be taken. However, the Viticulture Research Department has used Landsat 8 imagery, and switching to a new satellite system with a different spatial resolution would drastically change the Sample Locator Tool's methods. Currently, the clients decided that the methods should be tested with Landsat 8 imagery before any edits to the methods were changed. In the future it would be interesting to adjust these methods to test how well Sentinel 2 imagery could identify sample locations.

\subsection{Summary}

The planning, implementation, and analyses involved in this project were applied to determine if satellite imagery could identify sample locations used to estimate vineyard yield. This chapter summarized the results used to address this inquiry. From the yield estimation tables presented in Section 6.1, it is clear that the methods used in the Sample Locator Tool did not identify optimal sample locations using the NDVI and TVI imagery. For the 2017 and 2018 harvest seasons, the Random 20 Point sample estimated yield more accurately than the other tested categories, which suggests that improvements need to be made to the methods used in the Sample Locator Tool. Within Section 6.2, two possible limitations present in this project were discussed. Overall, the results and analyses presented in this chapter suggest that further adjustments to the Sample Locator Tool are required before it can be implemented on a larger scale. 


\section{Chapter 7 - Conclusions and Future Work}

This chapter represents the completion of this project. Section 7.1 offers a summary and conclusion for the planning, methods, and results that developed through this project. The final passage of this report is Section 7.2, which discusses possible future work that can be implemented to further the research that has been presented.

\subsection{Summary and Conclusion}

This project developed from the Viticulture Research Department's interest in applying a technique currently used for grape maturity sampling and instead applying the same technique to estimate vineyard yield. Through this interest, the goal of the project was to determine if satellite imagery could identify accurate sampling locations to estimate yield. Three objectives were set to measure the progress of the project and to ensure the goal was met.

To achieve the goal set by the Viticulture Research Department, the first objective was to analyze the relationship between the yield maps, NDVI data, and TVI data. The relationships between yield and the vegetation indices were evaluated using pixel-bypixel correlation analyses. It was important to quantify this relationship to determine what months of the Landsat 8 imagery should be used and if either vegetation index had a stronger linear correlation with the yield maps. The results from this analysis established that imagery from July and August had the strongest correlations with the yield maps and that neither vegetation index performed significantly better than the other. Therefore, NDVI and TVI imagery derived from July and August Landsat 8 rasters were used to output the sample location files.

The second objective was to develop a tool that processes satellite imagery to output optimal sample locations used to estimate yield. To meet this requirement the Sample Locator Tool was created to function similarly to the methods documented in Meyers et al. (2020). This tool iterated through a folder of resampled yield maps or remote imagery and analyzed the rasters three consecutive pixels at a time, based on the vine angle of the vineyard block. The location of the sample was chosen based on the mean of three pixels that matched the mean raster value of the entire block. The result of the Sample Locator Tool was sets of sample locations derived from the resampled yield maps, NDVI imagery, and TVI imagery for several years.

The final objective of the project was to determine the effectiveness of each sample location by comparing the yield estimation errors between the sample location categories. The yield estimation errors were measured by calculating the difference between the sample location's yield estimation and the mean yield of its corresponding yield map. Paired t-tests were used to evaluate the statistical difference between the yield estimations derived from the sample location categories. The results of the paired t-tests determined that the Random 20 Points sample file provided the most accurate yield estimations. Therefore, the samples generated from the Sample Locator Tool were not as accurate as the random samples. However, future work could be explored to improve the methods that were tested in this project. 


\subsection{Future Work}

There are multiple components that could be implemented to continue the research presented in this report. The first and most important component is the addition of field sampling. The second addition that should be highly considered is the alteration of the Sample Locator Tool's methods. The third adjustment that could be implemented is the inclusion of climate and vineyard metric datasets. Finally, the creation of the Landsat boundary file will need to be adjusted if similar methods of the Sample Locator Tool are used on a larger scale. Together, these four components are the most important additions that should be addressed in the future.

One of the key elements missing from this project is some form of field sampling. As mentioned in the previous chapter, field sampling is utilized in a majority of viticulture research reports. Although the time frame of this project prohibited the use of field sampling, the yield maps provided by the Viticulture Research Department were a good substitute to quantify the variability of yield in the vineyard. However, it is recommended that further research involving the methods in this project should include field sampling to verify the results.

The second aspect of the project that could be addressed is the alteration of the Sample Locator Tool's methods. It is possible that determining the location of the three pixels based solely on the mean value of the raster is not ideal. In the future other methods should be tested, such as using the standard deviation as a measure instead of the mean. Another approach that could be tested is directly using the methods described in Meyers et al. (2020), by finding three pixels that closely match the three quantile means of the entire vineyard block. Ultimately, another method of pixel identification will be required to produce more accurate results.

The third component that could be focused on is the implementation of other sources of data. According to Dami and Sabbatini (2011), inconsistent weather across harvest seasons can result in inconsistent vineyard yield. Weather plays an important role in the maturation of wine grapes, thus implementing weather data in the methods of the Sample Locator Tool would be an interesting approach. Another dataset that could be analyzed are soil maps. Research has shown that soil variability is a major contributor to the variability of vineyard characteristic, which include yields (Bramley \& Lamb, 2003). Therefore, soil data would provide another point of analysis that could possibly improve yield estimation results. A third dataset that could be included in future research is Sentinel 2 imagery. It provides higher resolution imagery than Landsat 8, which could provide more precise sample locations than the ones presented in this project. Overall, these three datasets should be considered to check if improvements can be made to the Sample Locator Tool described in this project

The final component of this project that would need to be addressed is the creation of the Landsat boundary file that is described in Section 4.4.1. Currently, the process of creating these boundary files for each individual vineyard block is time-consuming. For the study area of this project, creating a boundary file for each block was straightforward. However, it is unlikely that the creation of a Landsat boundary file on a commercial scale would be reasonable. Ultimately, these are four additions that would be recommended for future work regarding the research presented in this project. 


\section{Works Cited}

Arnó, J., Martínez-Casasnovas, J.A., Ribes-Dasi, M., \& Rosell, J.R. (2009) Review. Precision viticulture. Research topics, challenges and opportunities in site-specific vineyard management. Spanish Journal of Agricultural Research, 2009, vol. 7, num. 4, p. 779-790.

Bramley, R. G. V., \& Hamilton, R. P. (2004). Understanding variability in winegrape production systems: 1 . Within vineyard variation in yield over several vintages. Australian Journal of Grape and Wine Research, 10(1), 32-45.

Bramley, R. G. V. \& Lamb, D. (2003) Making sense of vineyard variability in Australia. Proceedings of an international symposium on Precision Viticulture, Ninth Latin American Congr. on Viticulture and Oenology, 35-54.

Bramley, R. G. V., \& Lanyon, D. M. (2002). Evidence in support of the view that vineyards are leaky-Indirect evidence and food for thought from precision viticulture research. In: Vineyard 'leakiness'. Proceedings of a workshop held at the Waite Campus, Adelaide, January 24-25, 2002, to scope the potential threat to the sustainability of Australian viticulture through excessive drainage below the root zone. Final Report on GWRDC Project No. GWR01/04. (CSIRO Land and Water / Grape and Wine Research and Development Corporation, Adelaide).

Bramley, R. G. V., \& Proffitt, A. P. B. (1999). Managing variability in viticultural production. Grapegrower and Winemaker, 427, 11-16.

Broge, N. H., \& Leblanc, E. (2000). Comparing prediction power and stability of broadband and hyperspectral vegetation indices for estimation of green leaf area index and canopy chlorophyll density. Remote sensing of environment, 76, 156172.

Cunha, M., Marcal, A.R.S., \& Silva, L. (2010) Very early prediction of wine yield based on satellite data from VEGETATION. International Journal of Remote Sensing 31, 3125-3142.

Dami, I. \& P. Sabbatini. 2011. Crop Estimation of Grapes. The Ohio State University Fact Sheet HYG1434-11.

Gamon, J. A., Field, C. B., Goulden, M. L., Griffin, K. L., Hartley, A. E., Joel, G., Penuelas, J., \& Valentini, R. (1995). Relationships between NDVI, canopy structure, and photosynthesis in three Californian vegetation types. Ecological Applications, 5(1), 28-41.

Hall, A., Lamb, D., Holzapfel, B., \& Louis, J. (2002) Optical remote sensing applications in viticulture-a review. Australian Journal of Grape and Wine Research 8, 36-47. 
Hall, A., \& Wilson, M. A. (2013). Object-based analysis of grapevine canopy relationships with winegrape composition and yield in two contrasting vineyards using multitemporal high spatial resolution optical remote sensing. International Journal of Remote Sensing, 34(5), 1772-1797.

Komm, B., \& Moyer, M. (2015). Vineyard Yield Estimation. Washington State University Extension Bulletin EM086E.

Li, Z., Yan, F. L., \& Fan, X. T. (2005). The variability of NDVI over Northwest China and its relation to temperature and precipitation. Journal of Remote Sensing, 9(3), 308.

Liu, S., Marden, S., \& Whitty, M. (2013). Towards automated yield estimation in viticulture. In Proceedings of the Australasian Conference on Robotics and Automation, Sydney, Australia (Vol. 24, pp. 2-6).

Meyers, J. M., Dokoozlian, N., Ryan, C., Bioni, C., \& Vanden Heuvel, J. E. (2020). A New, Satellite NDVI-Based Sampling Protocol for Grape Maturation Monitoring. Remote Sensing, 12(7), 1159.

Meyers, J. M., \& Vanden Heuvel, J. E. (2014). Use of Normalized Difference Vegetation Index Images to Optimize Vineyard Sampling Protocols. American Journal of Enology and Viticulture, 65(2), 250-253.

Openshaw, S., \& Taylor, P. (1979). A million or so correlation coefficients, three experiments on the modifiable areal unit problem. Statistical Applications in the Spatial Sciences, 127-144.

Rouse, J. W., Haas, R. H., Schell, J. A., \& Deering, D. W. (1974). Monitoring vegetation systems in the Great Plains with ERTS. NASA special publication, 351, 309.

Sanchez, L. A., Sams, B., Alsina, M. M., Hinds, N., Klein, L. J., \& Dokoozlian, N. (2017). Improving vineyard water use efficiency and yield with variable rate irrigation in California. Advances in Animal Biosciences, 8(2), 574-577.

Schumann, A. W. (2010). Precise placement and variable rate fertilizer application technologies for horticultural crops. HortTechnology, 20(1), 34-40.

Sun, L., Gao, F., Anderson, M., Kustas, W., Alsina, M., Sanchez, L., Sams, B., McKee, L., Dulaney, W., White, W., Alfieri, J., Prueger, J., Melton, F., \& Post, K. (2017). Daily Mapping of $30 \mathrm{~m}$ LAI and NDVI for Grape Yield Prediction in California Vineyards. Remote Sensing, 9(4), 317-335.

Tisseyre, B., Ojeda, H., \& Taylor, J. (2007). New technologies and methodologies for sitespecific viticulture. Journal International des Sciences de la Vigne et du Vin, 41(2), 63-76. 


\section{Appendix A. Sample Locator Tool}

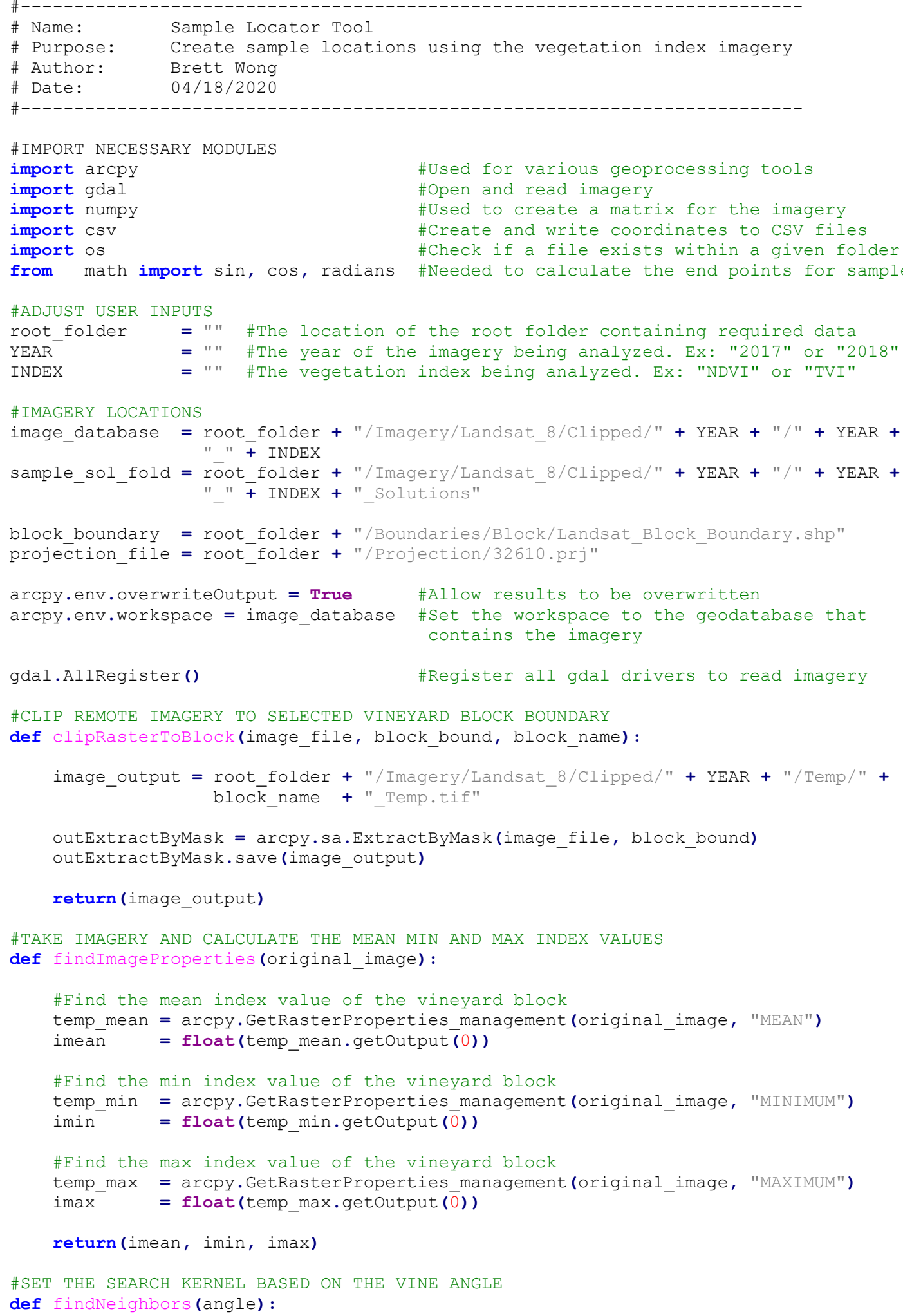




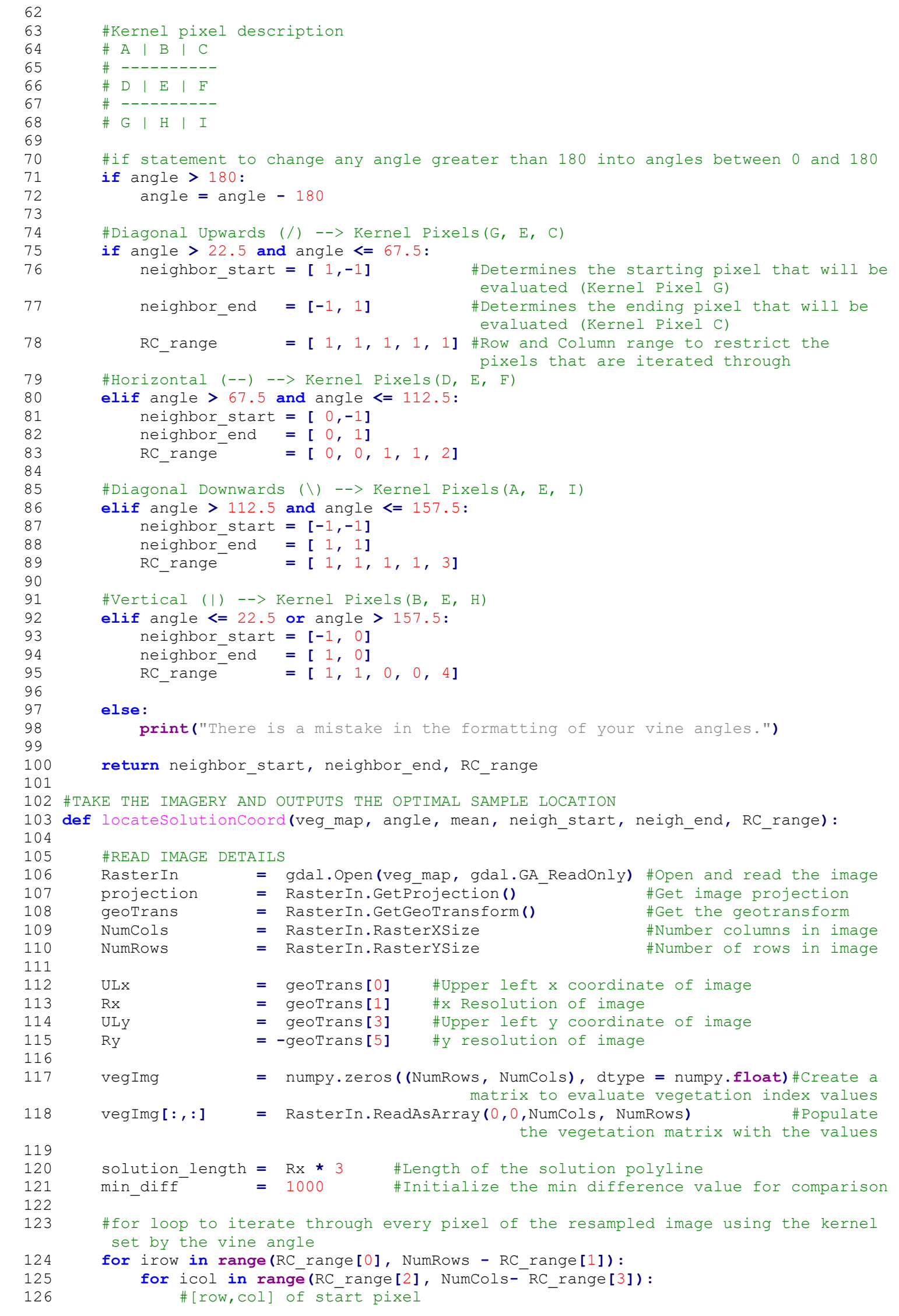




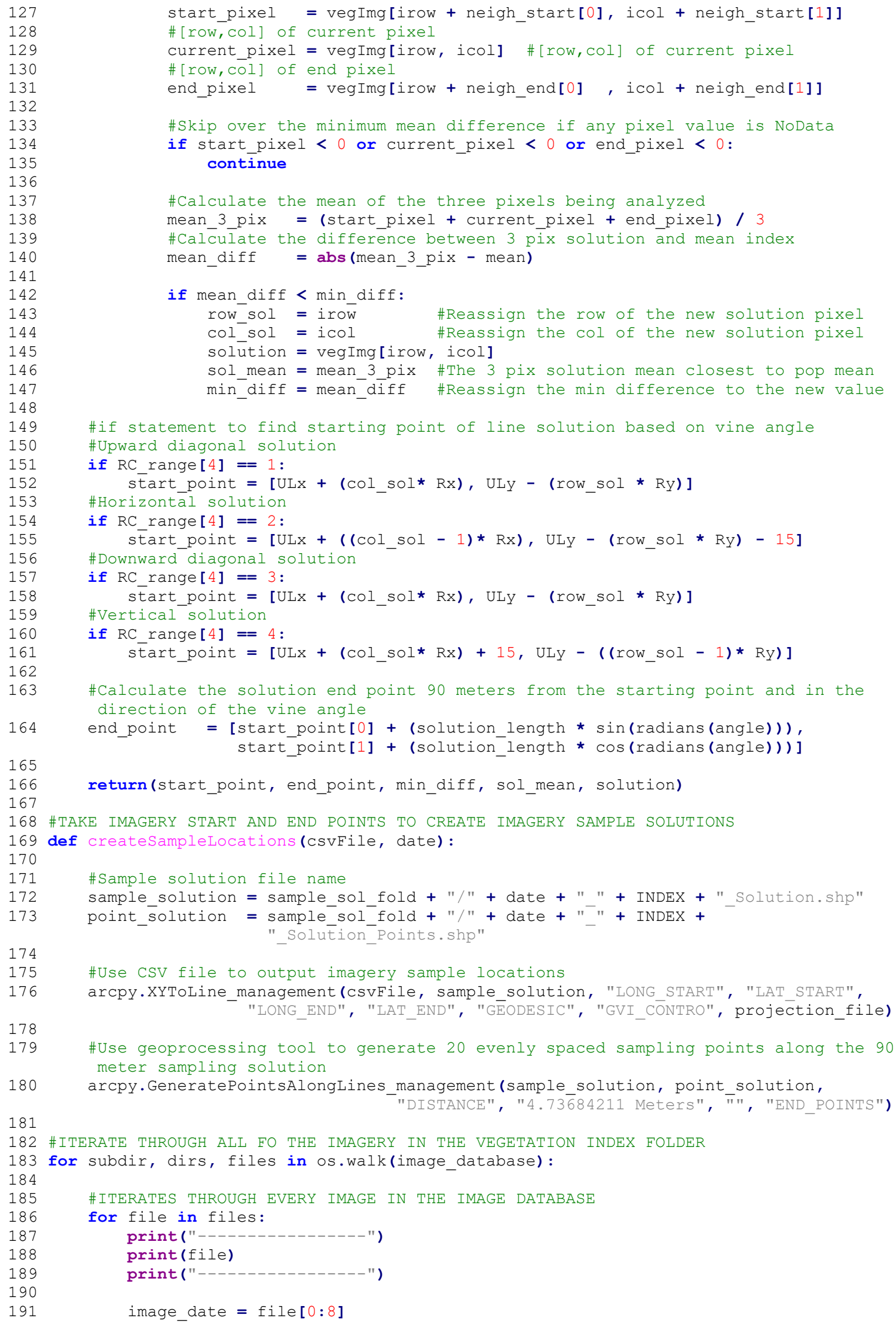




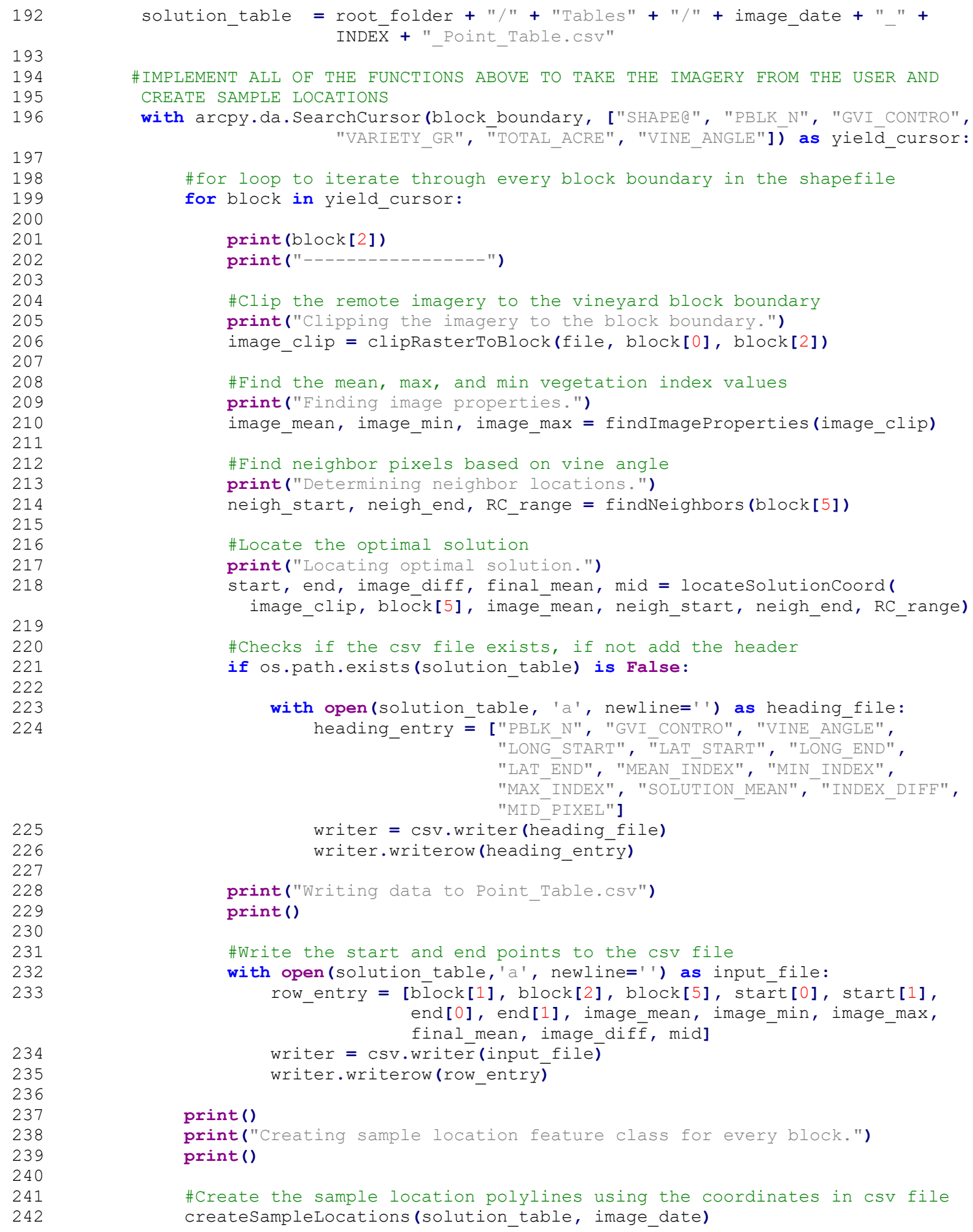

with open(solution_table, 'a', newline='') as heading_file: heading_entry $\overline{=}[$ "PBLK_N", "GVI_CONTRO", "VINE_ANGLE", "LONG START", "LAT START", "LONG END",

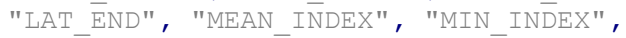




\section{Appendix B. Yield Extraction Tool}

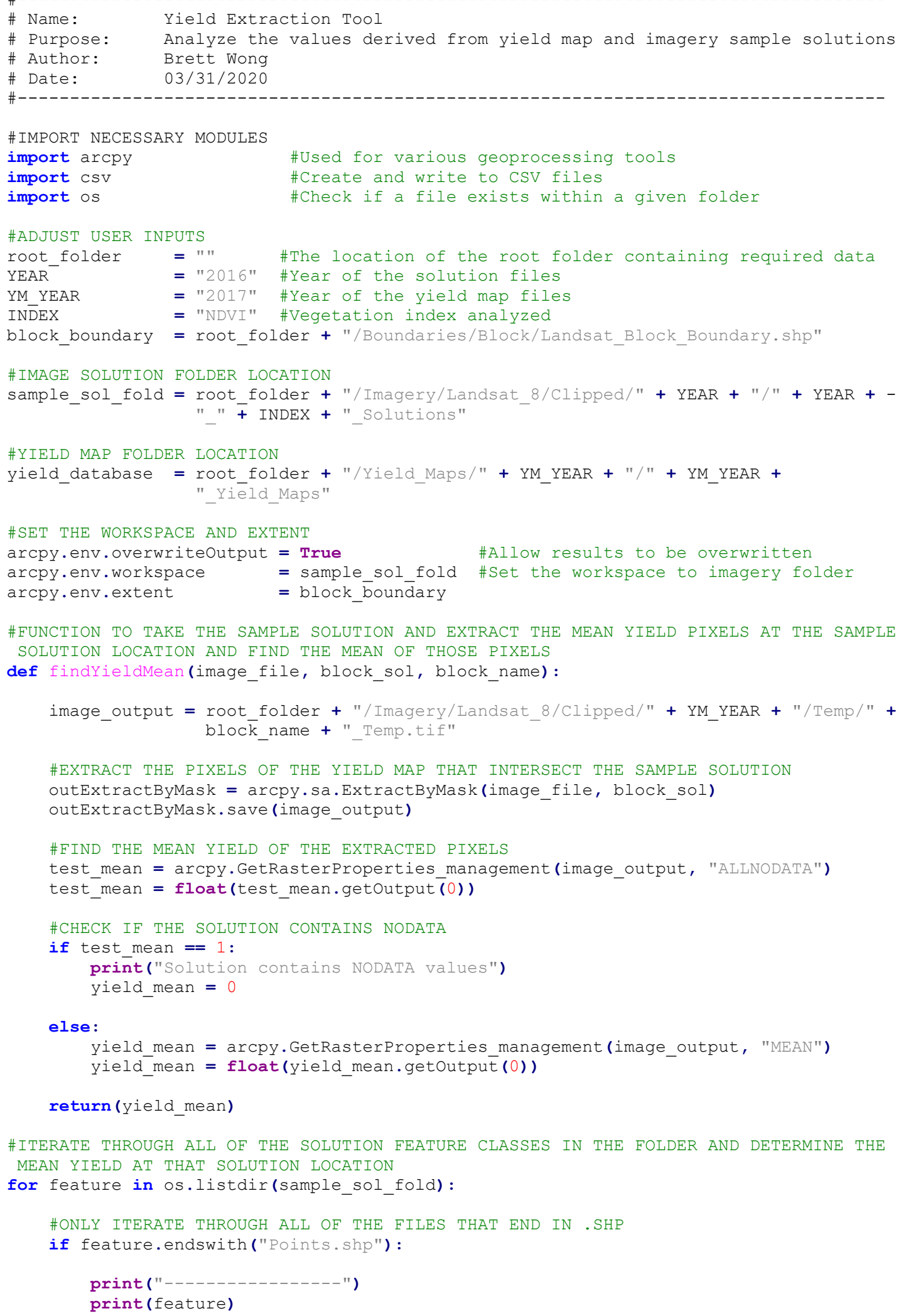




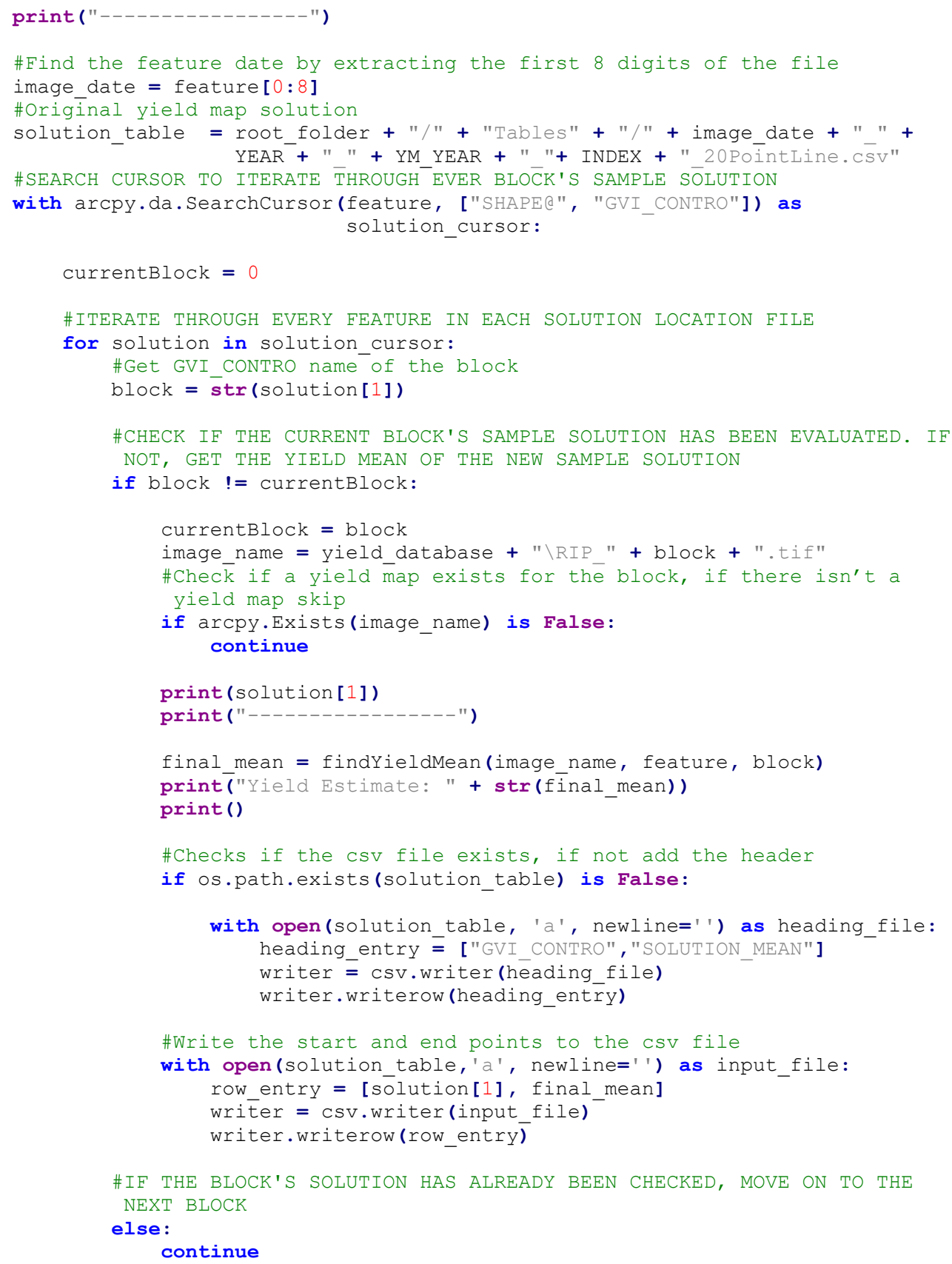

